PHYSICAL REVIEW D 81, 103002 (2010)

\title{
Dynamical masses in modified gravity
}

\author{
Fabian Schmidt \\ Theoretical Astrophysics, California Institute of Technology M/C 350-17, Pasadena, California 91125-0001, USA
}

(Received 10 March 2010; published 7 May 2010)

\begin{abstract}
Differences in masses inferred from dynamics, such as velocity dispersions or $\mathrm{x}$ rays, and those inferred from lensing are a generic prediction of modified gravity theories. Viable models, however, must include some nonlinear mechanism to restore general relativity (GR) in dense environments, which is necessary to pass Solar System constraints on precisely these deviations. In this paper, we study the dynamics within virialized structures in the context of two modified gravity models, $f(R)$ gravity and Dvali-GabadadzePorrati (DGP). The nonlinear mechanisms to restore GR, which $f(R)$ and DGP implement in very different ways, have a strong impact on the dynamics in bound objects; they leave distinctive signatures in the dynamical mass-lensing mass relation as a function of mass and radius. We present measurements from $N$-body simulations of $f(R)$ and DGP, as well as semianalytical models that match the simulation results to surprising accuracy in both cases. The semianalytical models are useful for making the connection to observations. Our results confirm that the environment and scale dependence of the modified gravity effects have to be taken into account when confronting gravity theories with observations of dynamics in galaxies and clusters.
\end{abstract}

DOI: $10.1103 /$ PhysRevD.81.103002

PACS numbers: 95.30.Sf, 04.50.Kd, 95.36.+x, 98.80.Jk

\section{INTRODUCTION}

Gravity, as described by general relativity (GR), is remarkably weakly constrained in the present day on scales larger than a few astronomical units. Though measurements from binary pulsar timing to the cosmic microwave background $(\mathrm{CMB})$ and big bang nucleosynthesis are all consistent with GR, there is still room for order unity deviations in the cosmos today, on scales of kpc and larger. Thus, testing gravity on cosmological scales is an interesting frontier and the focus of much current research [1-11].

Any gravity theory that attempts to be complete has to satisfy stringent Solar System constraints and has to locally match the predictions of GR to within one part in $10^{5}$ there. Only a few consistent models that modify gravity appreciably on large scales, but restore GR locally, are known. Two of them will be the subject of this study: $f(R)$ gravity [12-15] and the DGP model [16]. Within certain bounds placed by the CMB and expansion history measurements in addition to Solar System tests, both theories can be made to satisfy all current constraints on gravity (including the observation of an accelerating expansion). In both models there exists a nonlinear mechanism to restore GR in highdensity environments: the chameleon effect for $f(R)$, and the Vainshtein mechanism for DGP. Furthermore, all currently known consistent modifications of gravity on large scales include some variant of either of these mechanisms. In order to be able to constrain these models with cosmological data, it is crucial to correctly include the nonlinear mechanisms. Recently, $N$-body simulations of $f(R)$ [17] and DGP [18-20] have been done, which self-consistently solve the nonlinear field equations together with the growth of structure (see also [21] for the first study of the DGP case, using a different approach). In principle, it has be- come possible with these simulations to unlock the wealth of observations available on nonlinear scales to probe gravity, albeit in a necessarily model-dependent way.

It is well known that the additional degrees of freedom present in modified gravity theories generically affect the dynamical potential, which governs the propagation of nonrelativistic bodies, differently than the lensing potential, which governs the propagation of massless particles such as light (e.g., [22]). Thus, comparing dynamical with lensing mass estimates is an interesting and quite generic probe of modifications to gravity. In this paper, we study the signatures of $f(R)$ and DGP in dynamical observables such as velocity dispersions, compared to lensing, which measures essentially the "true" mass (i.e. the integral over the rest-frame density) in both models.

Constraints on the difference between dynamical and lensing potential are often phrased in terms of the postNewtonian parameter $\gamma_{\text {PPN }}$ [Eq. (5) below], in analogy to Solar System tests. In general, however, the departures from GR cannot be encapsulated by a single parameter but are functions of scale, time, and the local environment. In particular, this is the case for both $f(R)$ and DGP. Hence, we introduce a more generally applicable quantity $\mathfrak{g}$ [Eq. (3)], which is defined directly via the modified forces, and is well suited for predictions in the context of $f(R)$ and DGP as well as for constraints from observations.

Velocities of extragalactic objects are measured through their redshifts $z$, which receive a contribution $|\Delta z|=v_{\|} / c$ from the line-of-sight velocity $v_{\|}$. In the cosmological context, there are two regimes where the dynamics of matter can be understood fairly easily: on very large scales, linear perturbation theory in the matter density is valid, simplifying the theoretical predictions. Large-scale velocity fields can be measured through the redshift distortion of 
the power spectrum, which thus offers a probe of the dynamical potential $[23,24]$. On small scales, most of the observable matter lies in gravitationally bound dark matter halos. In this regime, for relaxed systems, the velocity distribution of collisionless objects such as dark matter, galaxies, or stars is related to the dynamical potential by the virial theorem. For collisional particles such as diffuse gas, this relation is given by hydrostatic equilibrium. The virial or thermal velocities can be observed as velocity dispersion of stars in galaxies, galaxies in clusters, or as $\mathrm{x}$-ray or Sunyaev-Zeldovich signal from diffuse gas in clusters. Also, the redshift-space matter power spectrum on small scales is a probe of virial velocities [23,25].

This paper is concerned with the latter regime, and our goal is to study the dynamics of matter in halos. Since these are highly nonlinear systems, rigorous results can be obtained only via $N$-body simulations. We therefore present measurements from the modified gravity simulations of $f(R)$ and DGP $[17,18,20]$. However, for many practical purposes including comparison with observations, it is necessary to go beyond the simulation results that have limited resolution and cover only a few points in the parameter space of the models. Thus, a sufficiently accurate semianalytic model of the dynamics in modified gravity is desirable to bridge the gap with observations.

Fortunately, we can make some justified assumptions that simplify the problem greatly: first, since we are concerned with subhorizon scales, we employ the quasistatic approximation, neglecting time derivatives and assuming the halos are in steady state. Further, we assume spherically symmetric halos. While certainly not realistic, deviations from spherical symmetry are not expected to affect the results qualitatively. Throughout, we will assume a Navarro-Frenk-White (NFW) [26] profile, although all derivations can easily be generalized to different profiles. The problem is then reduced to finding the solution of the field equations for a spherically symmetric mass and calculating the modified gravitational force. The accuracy of this simplified model can then be benchmarked with the simulation results.

The paper is structured as follows. In Sec. II, we introduce our main observable, the modified gravitational force strength, and present the theoretical expectations and semianalytic models for $f(R)$ and DGP. Section III contains the simulation results and comparisons with the theoretical models. We then discuss the application to observations in Sec. IV. We conclude in Sec. V.

\section{THEORETICAL EXPECTATIONS}

In this section, we derive theoretical expectations for the modified gravitational forces and virial quantities measured in the simulations in Sec. III and connected to observations in Sec. IV. Gravitational forces are given by the gradient of the dynamical potential $\Psi$, defined via the perturbed Friedmann-Robertson-Walker metric in
Newtonian gauge:

$$
d s^{2}=-(1+2 \Psi) d t^{2}+a^{2}(t)(1+2 \Phi) d \mathbf{x}^{2} .
$$

As a reference point, we consider GR in the Newtonian limit, where the dynamical potential satisfies the Poisson equation

$$
\nabla^{2} \Psi=\nabla^{2} \Psi_{N}=4 \pi G \delta \rho,
$$

where $\delta \rho=\rho / \bar{\rho}$ is the total matter overdensity. Assuming spherical symmetry, which we will throughout, we can define a parameter $\mathrm{g}$ :

$$
\mathfrak{g}(r) \equiv \frac{d \Psi / d r}{d \Psi_{N} / d r},
$$

which quantifies the strength of the gravitational force in modified gravity relative to that which would be measured in GR given the same density field. $g=1$ corresponds to unmodified forces. Here we have suppressed the dependence of $g$ on the scale factor $a$; unless otherwise stated, we will always assume $a=1$.

In the models we consider, the lensing potential satisfies $^{1}$

$$
\Psi_{-} \equiv \frac{1}{2}(\Psi-\Phi)=\Psi_{N} .
$$

Hence, $\mathfrak{g}$ can be probed, for example, by comparing dynamical to lensing mass estimates of a given object. Such comparisons in the Solar System [27] or for distant galaxies [28] are often phrased in terms of the post-Newtonian parameter $\gamma_{\mathrm{PPN}}$ :

$$
\gamma_{\mathrm{PPN}}=-\frac{\Phi}{\Psi}=2 \frac{\Psi_{-}}{\Psi}-1 \stackrel{\mathrm{BD}}{=} 2 \mathfrak{g}^{-1}-1 .
$$

The last equality relating $\gamma_{\mathrm{PPN}}$ to our $\mathfrak{g}$ parameter [where we have used Eq. (4)] is valid only when the force modifications are scale independent, such as in Brans-Dicke (BD) type scalar-tensor theories. Note that the parametrized post-Newtonian (PPN) parameter is formally defined via the potentials, while our $\mathrm{g}$ parameter is derived in terms of forces. Only forces, or more generally derivatives of the potentials $\Psi, \Psi_{-}$are observable, and a specific solution of the potentials (e.g., the Schwarzschild metric) is used to infer $\gamma_{\text {PPN }}$. However, in the models we consider $g$ is generally scale dependent; i.e. the scalar degrees of freedom do not follow the same scaling with distance as the GR potentials. Hence, it is advantageous to define a parameter based directly on the forces, rather than $\gamma_{\mathrm{PPN}}$, which is not immediately linked to observables.

In many practical cases, one is interested in a weighted average of $g$ over an object or region of space,

\footnotetext{
${ }^{1} \operatorname{In} f(R)$, there are corrections of order $\bar{f}_{R}$, and $\left|\bar{f}_{R}\right| \leq\left|f_{R 0}\right| \leq$ $10^{-4}$ for the models we consider; this is negligible compared to the $O(0.1)$ effects we will discuss.
} 


$$
\overline{\mathfrak{g}}_{w}=\frac{\int r^{2} w(r) \mathfrak{g}(r) d r}{\int r^{2} w(r) d r},
$$

where $w$ is a weighting function depending on the precise observable considered (we will turn to this in Sec. IV). The key point is that given a prediction for $g(r)$ we can estimate any such weighted average (as long as spherical symmetry is a sufficiently good approximation). In the next section, we will introduce one such averaged force modification that is relevant for comparison with simulations. We will then review the Newtonian potential and scaling relations for a dark matter halo with a NFW profile, before studying the same case for $f(R)$ and DGP gravity.

\section{A. Virial theorem and velocity dispersion}

For the comparison with our dark matter-only simulations, it is useful to consider a collisionless system in virial equilibrium. In that case, the virial theorem states that

$$
\begin{gathered}
W=-2 T, \quad \text { where } \\
W \equiv-\int d^{3} \mathbf{x} \rho(\mathbf{x}) \mathbf{x} \cdot \nabla \Psi(\mathbf{x}), \\
T \equiv \frac{1}{2} \int d^{3} \mathbf{x} \rho(\mathbf{x}) \sigma_{v, 3 D}^{2}(\mathbf{x}),
\end{gathered}
$$

denote the trace of the potential energy tensor and potential energy, respectively. Here $\sigma_{v, 3 D}^{2}=3 \sigma_{v, 1 D}^{2}$ is the threedimensional velocity dispersion (see Eq. (57) for our practical definition in terms of dark matter particles). Since the virial theorem is derived from the collisionless Boltzmann equation, and is thus a consequence of energy-momentum conservation, it is unchanged in any metric theory of gravity, and hence also in the models we consider. The modification enters through the modified relation between the potential $\Psi$ and the matter distribution.

Note that in the cosmological context, we are not dealing with strictly isolated systems, so that Eq. (7) does not hold precisely. Nevertheless, the validity of $W=\alpha T$ for simulated dark matter halos has been shown to hold to high accuracy [29-31]. Here, the constant $\alpha$ depends on the mass and radius definition chosen for the halos.

In the spherically symmetric case, we can use the definition of $\mathfrak{g}$ [Eq. (3)] to relate the potential energy tensor and kinetic energy in modified gravity to the Newtonian values $W_{N}, T_{N}$ :

$$
\frac{W_{\text {mod.gr }}}{W_{N}}=\frac{T_{\text {mod.gr }}}{T_{N}}=\overline{\mathrm{g}}_{\mathrm{vir}},
$$

where $\overline{\mathfrak{g}}_{\text {vir }}$ is given by Eq. (6) with a weighting function

$$
w_{\text {vir }}(r)=\rho(r) r \frac{d \Psi_{N}}{d r} .
$$

The gradient of the Newtonian potential appearing here is uniquely determined by the density $\rho(r)$, assuming that external tidal fields are negligible.

\section{B. Newtonian potential of a halo}

Let us consider the GR case first. We can integrate Eq. (2) to obtain

$$
\begin{gathered}
\frac{d \Psi_{N}}{d r}=\frac{G \delta M(<r)}{r^{2}}, \\
\delta M(<r) \equiv 4 \pi \int_{0}^{r} d r^{\prime} r^{\prime 2} \delta \rho\left(r^{\prime}\right) .
\end{gathered}
$$

Note that $\delta M$ is defined in terms of the enclosed overdensity $\delta \rho$. Imposing the condition $\Psi_{N}(r \rightarrow \infty)=0$, we can integrate again and obtain

$$
\Psi_{N}(r)=-\int_{r}^{\infty} d r^{\prime} \frac{G \delta M\left(<r^{\prime}\right)}{r^{\prime 2}} .
$$

Let us now consider an NFW halo with mass $M_{\Delta}$, defined as the mass contained within a radius $R_{\Delta}$ so that the average density within $R_{\Delta}$ is $\bar{\rho} \Delta$ (note that $\Delta$ here is arbitrary and does not have to correspond to a certain "virial" overdensity). The NFW profile has been shown to be a good match even to the halos in modified gravity simulations $[20,32]$. We define the corresponding concentration as $c_{\Delta}=R_{\Delta} / r_{s}$. We will consider an untruncated profile here; while this overestimates the exterior mass somewhat, it is closer to the profiles measured in simulations than the other simple choice, a truncated profile. Then, the density profile is given by

$$
\begin{aligned}
& \rho(r)=4 \rho_{s} f_{\mathrm{NFW}}\left(r / r_{s}\right), \\
& f_{\mathrm{NFW}}(y)=\frac{1}{y(1+y)^{2}},
\end{aligned}
$$

where $\rho_{s}=\rho\left(r_{s}\right)$ is chosen so that the mass within $R_{\Delta}$ is $M_{\Delta}$, and we have

$$
\begin{gathered}
\delta M(<r)=M_{\Delta} \frac{F\left(c_{\Delta} r / R_{\Delta}\right)}{F\left(c_{\Delta}\right)}\left[1-\frac{F\left(c_{\Delta}\right)\left(r / R_{\Delta}\right)^{3}}{F\left(c_{\Delta} r / R_{\Delta}\right) \Delta}\right], \\
F(y)=-\frac{y}{1+y}+\ln (1+y) .
\end{gathered}
$$

The correction in square brackets in Eq. (17) is usually neglected since it is smaller than $\Delta^{-1}$, and we will do so here as well in order to simplify the analytical expressions. From this, we get

$$
\frac{d \Psi_{N}}{d r}=\frac{\Psi_{\Delta}}{R_{\Delta}} \frac{R_{\Delta}^{2}}{r^{2}} \frac{F\left(c_{\Delta} r / R_{\Delta}\right)}{F\left(c_{\Delta}\right)},
$$

where the potential scales with $\Psi_{\Delta}$ defined by

$$
\Psi_{\Delta} \equiv \frac{G M_{\Delta}}{R_{\Delta}} .
$$


We can integrate to obtain the potential for an isolated NFW halo:

$$
\begin{gathered}
\Psi_{N}(r)=-\Psi_{\Delta} E\left(\frac{r}{R_{\Delta}}, c_{\Delta}\right), \\
E(x, c) \equiv \frac{(1+c) \ln (1+c x)}{(1+c) x \ln (1+c)-c x} .
\end{gathered}
$$

$E(0, c) \sim 5-12$ (for $c \sim 4-30$ ) gives the central depth of the potential well for an isolated NFW halo with concentration $c$ in units of $\Psi_{\Delta}$. Note that in reality, the depth of the potential well will depend on the large-scale environment, so that Eq. (21) will only give a rough scaling. $\Psi_{\Delta}$ in turn is given by

$$
\begin{gathered}
\Psi_{\Delta}=\left(G M_{\Delta} H_{0}\right)^{2 / 3}\left(\frac{1}{2} \Omega_{m} \Delta\right)^{1 / 3} \\
=\left(\frac{M_{\Delta}}{6.26 \times 10^{22} M_{\odot} / h}\right)^{2 / 3}\left(\frac{1}{2} \Omega_{m} \Delta\right)^{1 / 3} \\
=1.79 \times 10^{-5}\left(\frac{M_{\Delta}}{10^{15} M_{\odot} / h}\right)^{2 / 3},
\end{gathered}
$$

where for the last equality we have assumed $\Omega_{m}=0.25$ and $\Delta=200$. Note the scaling of $\Psi_{\Delta}$ with $M_{\Delta}^{2 / 3}$, because $M_{\Delta}$ and $R_{\Delta}$ are linked through the fixed overdensity $\Delta$. Throughout, unless otherwise stated, we use the concentration relation of [33]:

$$
c(M, z)=\frac{9}{1+z}\left(\frac{M}{M_{*}(z)}\right)^{-0.13} .
$$

Here, $M_{*} \approx 3.2 \times 10^{12} M_{\odot} / h$ for our fiducial $\Lambda$ CDM cosmology. Recently, more accurate expressions for the concentration have been found $[34,35]$. However, our results are not very sensitive to the concentration; hence we deem Eq. (26) sufficient. At the very highest masses $M_{\Delta} \gtrsim$ $10^{15} M_{\odot} / h$; however, Eq. (26) underpredicts the concentration significantly (e.g., $[35,36])$. As a simple remedy, we take $c=\max \{4, c(M)\}$ in place of $c(M)$ from Eq. (26).

Finally, the weighted $\mathfrak{g}(r)$ quantifying the modification to the potential and kinetic energy [Eq. (10)] can be written as

$$
\overline{\mathfrak{g}}_{\mathrm{vir}}=\frac{\int_{0}^{1} d x x F\left(c_{\Delta} x\right) f_{\mathrm{NFW}}\left(c_{\Delta} x\right) \mathfrak{g}\left(x R_{\Delta}\right)}{\int_{0}^{1} d x x F\left(c_{\Delta} x\right) f_{\mathrm{NFW}}\left(c_{\Delta} x\right)},
$$

where $x=r / R_{\Delta}$.

\section{C. $f(\boldsymbol{R})$}

$f(R)$ gravity (see [15] for a review) is a modified action theory where the Einstein-Hilbert Lagrangian $R / 16 \pi G$ is replaced with $[R+f(R)] / 16 \pi G$. Throughout this section $R$ denotes the Ricci scalar. $f(R)$ models correspond to scalar-tensor theories, where the scalar degree of freedom is given by $f_{R} \equiv d f / d R$ and mediates the relation between density and space-time curvature. In order for the theory to be stable under perturbations, it is necessary that $f_{R}<0$ [37].

In the smooth background, the scalar field assumes a value of $\bar{f}_{R} \equiv f_{R}(\bar{R})$, where $\bar{R} \propto H^{2}$ is the scalar curvature of the background. In this paper, we only consider models with $\left|\bar{f}_{R}\right| \leq 10^{-4}$ and will thus drop higher order terms in the $f_{R}$ field, which simplifies the expressions. In the quasistatic regime, the $f_{R}$ field and the dynamical potential are then determined from the density field by the following coupled equations:

$$
\begin{aligned}
& \nabla^{2} \delta f_{R}=\frac{1}{3}\left[\delta R\left(f_{R}\right)-8 \pi G \delta \rho\right], \\
& \nabla^{2} \Psi=\frac{16 \pi G}{3} \delta \rho-\frac{1}{6} \delta R\left(f_{R}\right) .
\end{aligned}
$$

Here, $\delta$ stands for perturbations from the background value: $\delta f_{R} \equiv f_{R}-\bar{f}_{R}$ and $\delta R \equiv R-\bar{R} . R$ and $\delta R$ are nonlinear functions of the field $f_{R}$; hence Eqs. (28) and (29) are difficult to solve in general. However, there are two limiting cases that can be solved easily.

First, consider the case where $\bar{f}_{R}$ is much larger than typical potential wells in the universe. In that case, $\delta f_{R}$ sourced by the right-hand side (rhs) of Eq. (28) is always much less than $\bar{f}_{R}$, and we can linearize the $\delta R$ term:

$$
\delta R \approx \frac{1}{f_{R R}(\bar{R})} \delta f_{R},
$$

where $f_{R R}=d^{2} f / d R^{2}$. Equation (28) then becomes an equation for a massive scalar field with $m_{f_{R}}^{-2} \equiv \lambda_{C}^{2}=$ $3 f_{R R}(\bar{R})$. We call the inverse mass $\lambda_{C}$ of the field in the background the Compton wavelength. In this limit, $\delta R \ll$ $\bar{R}$ on scales smaller than $\lambda_{C}$. Equation (29) then tells us that $\Psi=4 / 3 \Psi_{N}$; i.e. gravitational forces are increased by $4 / 3$ within the range of the $f_{R}$ field given by $\lambda_{C}$.

In the opposite limit, both terms on the rhs of Eq. (28) are much larger than the left-hand side (lhs) $\sim \bar{f}_{R} / r^{2}$ on the scales of interest. Since the field perturbation is limited in magnitude to be less than $\left|\bar{f}_{R}\right|, \delta f_{R}$ has to adjust itself so that the two terms on the rhs cancel to a high degree; in other words,

$$
\delta R\left(f_{R}\right) \approx 8 \pi G \delta \rho .
$$

Hence, the GR expression is restored, and Eq. (29) yields $\Psi=\Psi_{N}$ accordingly. This is called the chameleon regime [38].

In order to determine the transition between these two regimes, we consider the solution for a spherically symmetric mass. Formally, we can write the solution for $\delta f_{R}$ as

$$
\begin{gathered}
\delta f_{R}(r)=\frac{2}{3} \frac{G \delta M_{\mathrm{eff}}(<r)}{r}, \\
\delta M_{\mathrm{eff}}(r)=4 \pi \int_{0}^{r} d r^{\prime} r^{\prime 2} \delta \rho_{\mathrm{eff}}\left(r^{\prime}\right),
\end{gathered}
$$




$$
\delta \rho_{\text {eff }}(r)=\delta \rho(r)-\frac{\delta R(r)}{8 \pi G} .
$$

With these definitions, the modified dynamical potential satisfies

$$
\nabla^{2} \Psi=4 \pi G\left(\delta \rho+\frac{1}{3} \delta \rho_{\mathrm{eff}}\right) .
$$

Equations (33) and (34) state that $\delta M_{\mathrm{eff}} \leq \delta M$. If the perturbation $\delta f_{R}$ is small for all $r$ (which in general is only true far away from the body), we can neglect the $\delta R$ term in Eq. (34). Then, $\delta M_{\text {eff }}=\delta M$ and we have $\left|\delta f_{R}(r)\right|=2 / 3\left|\Psi_{N}\right|$. However, the maximal value $\delta f_{R}$ can achieve is $\left|\bar{f}_{R}\right|$, in which case the $f_{R}$ field becomes 0 . Thus, we arrive at the following condition:

$$
\left|f_{R 0}\right| \leq \frac{2}{3} \Psi_{N} .
$$

If the value of $\Psi_{N}$ for the body is larger than this, the field must enter the chameleon regime. Then, $\delta \rho_{\text {eff }}$ is nonzero only outside of the radius where Eq. (36) is met. $\delta M_{\text {eff }}$ is thus given by the mass outside of this radius, which can be thought of as forming a thin shell. For this reason, Eq. (36) is also called the thin-shell criterion. Since cosmological potentials range from $10^{-6}$ to $10^{-5}$, we expect that the chameleon mechanism will operate for background field values $\lesssim 10^{-5}$.

This general picture holds for any viable functional form of $f(R)$. However, in order to evaluate the effect on the dynamics quantitatively and to compare with the $N$-body simulations, we have to adopt a specific model. The functional form used in the simulations $[17,32,39]$ is the one of [37] with $n=1$, i.e.

$$
f(R)=-2 \Lambda \frac{R / R_{c}}{R / R_{c}+1},
$$

parameterized by the two constants $\Lambda$ and $R_{c}$. If the present-day background curvature $\bar{R}_{0}$ is much greater than $R_{c}$, which will be the case for the $f(R)$ models considered here, we can expand Eq. (37) to first order in $R_{c} / R$ and define a new parameter $f_{R 0}=f\left(\bar{R}_{0}\right)$ so that

$$
f(R)=-2 \Lambda-f_{R 0} \frac{\bar{R}_{0}^{2}}{R} .
$$

The first term supplies an effective cosmological constant yielding accelerated expansion of the background. The second term, controlled by $f_{R 0} \ll 1$, determines the departures from GR and yields corrections to the background expansion of order $f_{R 0}$. Since we will consider models with $\left|f_{R 0}\right| \leq 10^{-4}$, the background expansion is essentially indistinguishable from $\Lambda \mathrm{CDM}$. Taking the derivative of Eq. (38), we obtain the relation between the scalar field and the local curvature at the present day:

$$
f_{R}=f_{R 0} \frac{\bar{R}_{0}^{2}}{R^{2}}
$$

Furthermore, the Compton wavelength $\lambda_{C} \propto \sqrt{f_{R R}} \propto$ $R^{-3 / 2}$.

Simulations were performed for a range of background field values $\left|f_{R 0}\right|=10^{-6}, 10^{-5}, 10^{-4}$. From our discussion above, we expect that the chameleon mechanism will operate in the intermediate and small field cases, while it will be essentially absent for the large field $\left(10^{-4}\right)$. In addition to the $f(R)$ simulations, ordinary $\Lambda \mathrm{CDM}$ simulations were performed using the same expansion history and initial conditions. The cosmological parameters used in the simulations are summarized in Table I.

Given a density field such as that for an isolated NFW profile, one can solve Eq. (28) numerically. We have done so for the spherically symmetric case using a onedimensional relaxation algorithm (in fact, we solve for $u$ defined by $f_{R}=\exp (u)$ to avoid overshooting to $f_{R}>0$ [17]). While only an approximation of the physical reality, the spherically symmetric case allows for a much higher resolution (at much smaller computing time) than achievable in the full 3D cosmological simulations. The boundary conditions are given by $d f_{R} / d r=0$ at $r=0$, and $\delta f_{R}=0$ at the outer edge of the grid, chosen here to be $r_{\max }=$ $50 \mathrm{Mpc} / h$. We use 4096 equally spaced grid points in $r$. Once $\delta f_{R}$ is known, Eqs. (33)-(35) can be evaluated using $\delta R\left(\delta f_{R}\right)$, and the modified forces are given by

$$
\mathfrak{g}_{f(R)}(r)=1+\frac{1}{3} \frac{M_{\mathrm{eff}}(<r)}{M(<r)} .
$$

Figure 1 shows the "effective density" $\delta \rho_{\text {eff }}$, which sources the perturbation $\delta f_{R}$ to the field, for a halo of mass $2 \times 10^{14} M_{\odot} / h$ and different values of $f_{R 0}$. For large values of $\left|f_{R 0}\right| \geqslant 2 \times 10^{-5}$, the thin-shell condition is never met, so that $\delta \rho_{\text {eff }}=\delta \rho$ everywhere (except at very large $r$ where the field decays due to its finite $\lambda_{C}$ ). For smaller field values, we can see that a "thin shell" develops. For $\left|f_{R 0}\right|=10^{-5}$ it is quite broad, while it narrows considerably for a small field of $\left|f_{R 0}\right|=10^{-6}$.

TABLE I. Parameters of the simulated $f(R)$ and DGP cosmologies. For each model, GR simulations with identical expansion history and initial conditions were also performed.

\begin{tabular}{lcccc}
\hline \hline & $f(R)$ & sDGP & nDGP-1 & nDGP-2 \\
\hline$\Omega_{m}$ & 0.24 & 0.258 & 0.259 & 0.259 \\
$\Omega_{\Lambda}$ (eff.) & 0.76 & 0 & 0.741 & 0.741 \\
$\lg \left|f_{R 0}\right|$ & $-4,-5,-6$ & $\ldots$ & $\ldots$ & $\ldots$ \\
$r_{c}[\mathrm{Mpc}]$ & $\ldots$ & 6118 & 500 & 3000 \\
$\beta(a=1)$ & $\ldots$ & -1.15 & 1.21 & 2.25 \\
$H_{0}[\mathrm{~km} / \mathrm{s} / \mathrm{Mpc}]$ & 73.0 & 66.0 & 71.6 & 71.6 \\
$100 \Omega_{b} h^{2}$ & 2.23 & 2.37 & 2.26 & 2.26 \\
$n_{s}$ & 0.958 & 0.998 & 0.959 & 0.959 \\
$10^{9} A_{s}\left(0.05 \mathrm{Mpc}^{-1}\right)$ & 2.24 & 2.02 & 2.11 & 2.11 \\
$\sigma_{8}(\Lambda \mathrm{CDM})^{\mathrm{a}}$ & 0.796 & 0.657 & 0.789 & 0.789 \\
\hline \hline
\end{tabular}

${ }^{a}$ Linear power spectrum normalization today of a $\Lambda \mathrm{CDM}$ model with the same primordial normalization. 
FABIAN SCHMIDT

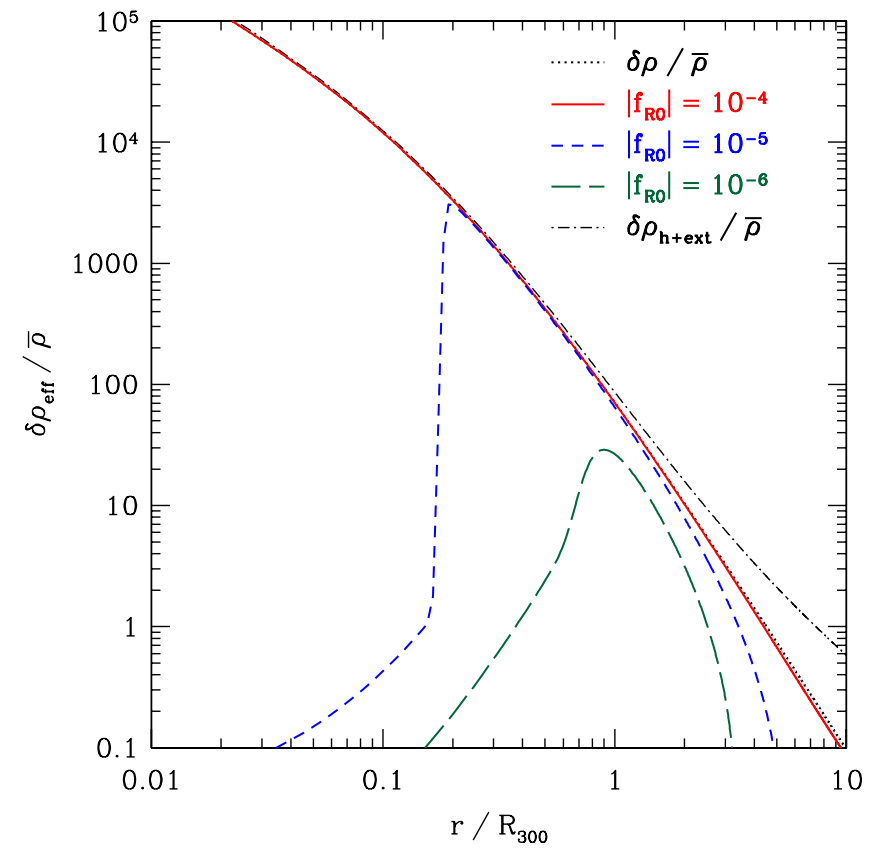

FIG. 1 (color online). $\delta \rho_{\text {eff }}$ [Eq. (34)] divided by the mean matter density $\bar{\rho}$, determined from the numerical solution of the $f(R)$ field equation for an NFW halo with $M_{300}=2 \times$ $10^{14} M_{\odot} / h$ for different values of $f_{R 0}$. Also shown is the matter density $\delta \rho / \bar{\rho}$ of the halo itself (dotted line almost matching the $10^{-4}$ field curve). The dash-dotted line shows a density profile, which matches that measured in simulations, including an additional external overdensity [Eq. (41)].

Note that the transition to $\rho_{\text {eff }}=0$ within the shell is very sharp, owing to the much smaller Compton wavelength within the body (recall $\lambda_{C} \propto R^{-3 / 2}$ ).

Figure 2 shows $\mathfrak{g}_{f(R)}(r)$ for $\left|f_{R 0}\right|=10^{-5}$ and different halo masses. The $10^{13} M_{\odot} / h$ halo is unscreeened, showing the $4 / 3$ force enhancement throughout. The $2 \times$ $10^{14} M_{\odot} / h$ halo is partially screened, while the $10^{15} M_{\odot} / h$ halo is screened to a large extent within $R_{300}$. For the latter two cases, we also indicate the screening radius $r_{\text {scr }}$ where, going from the outside in, the thin-shell condition Eq. (36) is first met. This radius serves as an indication of whether a given mass is screened, and roughly to what extent. As Eq. (36) shows, the screening radius depends on the depth of the potential well, which is influenced by the large-scale environment. To investigate this effect, we have added an external large-scale density field to the NFW profile, roughly matched to the halo profiles in our simulations [32] at large radii:

$$
\frac{\delta \rho_{\mathrm{h}+\mathrm{ext}}(r)}{\bar{\rho}}=\max \left\{\frac{\delta \rho_{\mathrm{NFW}}}{\bar{\rho}}, 30\left(\frac{r}{R_{\Delta}}\right)^{-1.46}\right\},
$$

where $\delta \rho_{\mathrm{NFW}}$ is the halo overdensity given by the NFW profile, and the external density is smoothly cut off at $\sim 40 \mathrm{Mpc} / h . \delta \rho_{\mathrm{h}+\mathrm{ext}}$ is shown as a dash-dotted line in Fig. 1. The resulting $\mathfrak{g}_{f(R)}$ including the external density
PHYSICAL REVIEW D 81, 103002 (2010)

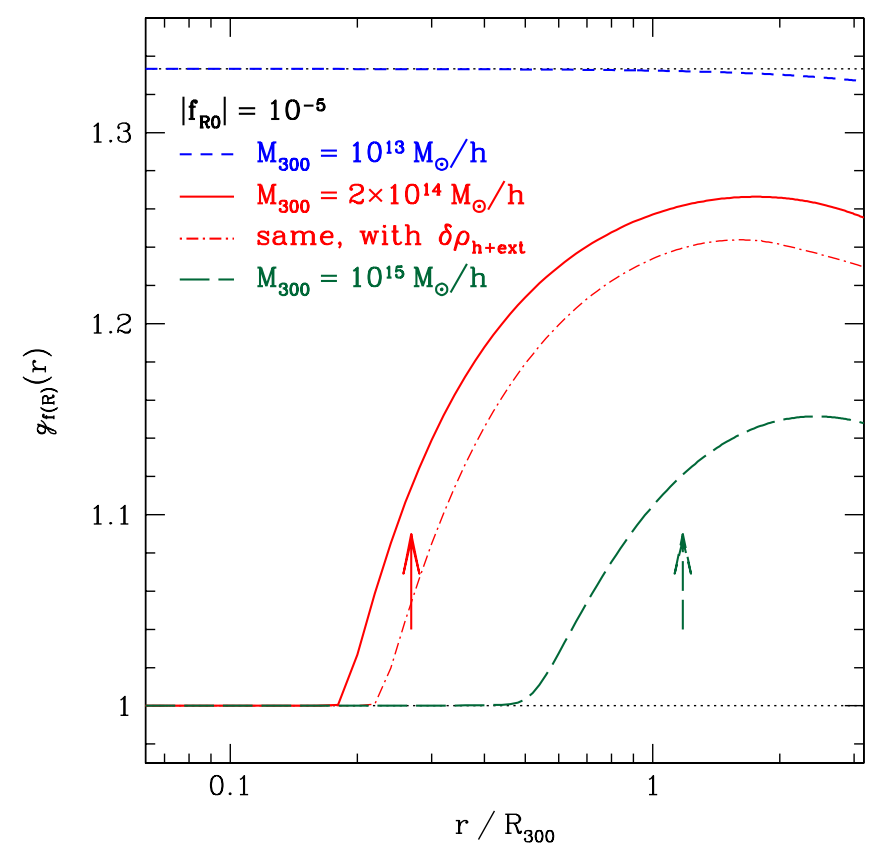

FIG. 2 (color online). $\mathfrak{g}_{f(R)}$ as a function of the scaled radius $r / R_{300}$ for $\left|f_{R 0}\right|=10^{-5}$ and different halo masses, from the numerical spherically symmetric solution. The low-mass halo is unscreened, showing the $4 / 3$ force enhancement throughout, while higher mass halos are partially screened. The arrows for the two more massive halos indicate at which $r$ the condition Eq. (36) is first met. For the $2 \times 10^{14} M_{\odot} / h$ halo, we also show $\mathfrak{g}_{f(R)}$ including an external density field [dash-dotted line; see Fig. 1 and Eq. (41)].

field is shown in Fig. 2 for the intermediate mass halo. Clearly, the field is screened at somewhat larger radii in this case, and $\mathfrak{g}_{f(R)}$ is smaller than that predicted for the NFW profile alone by about 0.04 in the transition region. Since halos can reside in a variety of environments, we expect significant scatter in the strength of the modified forces within halos in the $f(R)$ case, halos in overdense regions being screened more strongly than those in average or underdense regions. Further, we expect that the environment dependence will be more significant for lower mass halos than for massive halos $\left(\gtrsim 10^{14} M_{\odot} / h\right)$, since the former can be affected by a massive halo nearby, while the latter usually dominate their environment. We also investigated the effect of varying the halo concentration by $\pm 20 \%$; the impact on $\mathfrak{g}_{f(R)}$ is small in comparison with the effects of the large-scale environment, however.

Finally, using the results for $\mathfrak{g}_{f(R)}(r)$ we can evaluate Eq. (27). Figure 3 shows $\mathfrak{g}_{\mathrm{vir}, f(R)}$ as a function of mass for different values of the background field $f_{R 0}$. The thick lines and points show the numerical results from the relaxation code. For the strongest field, only the most massive halos (more massive than found in our limited volume simulations) are chameleon screened. For the weakest field, all halos above $M \sim 10^{13} M_{\odot} / h$ are expected to be screened, while for the intermediate field the transition scale is 


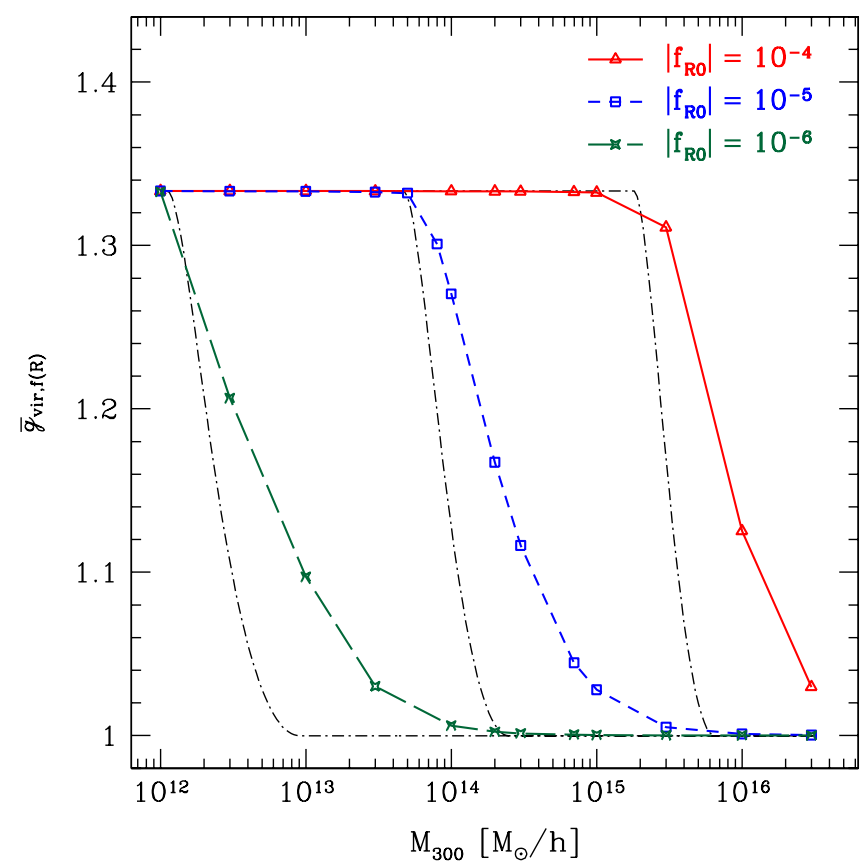

FIG. 3 (color online). Averaged modified gravitational force $\overline{\mathfrak{g}}_{\mathrm{vir}, f(R)}$ for NFW halos as a function of halo mass for different values of $\left|f_{R 0}\right|$. The points and thick lines show the numerical results from the $1 \mathrm{D}$ relaxation code. The thin black lines show the predictions of a simplified model Eq. (42). The behavior with mass is similar in the different models, with the transition mass between unscreened and screened regimes shifting as expected by simple estimates.

around $10^{14.5} M_{\odot} / h$, relevant for galaxy clusters. We will compare the predictions for both $\mathfrak{g}(r)$ and $\overline{\mathfrak{g}}_{\text {vir }}$ with simulation results in Sec. III.

As a simple analytic model for the numerical results, we make the assumption that all mass of the halo outside of $r_{\text {scr }}$ contributes to $\delta M_{\text {eff }}$. This results in the following simple prescription:

$$
\begin{aligned}
\mathfrak{g}_{f(R)}(r) & \approx 1+\frac{1}{3} \frac{M(<r)-M\left(<r_{\mathrm{scr}}\right)}{M(<r)} \\
& =1+\frac{1}{3}\left(1-\frac{F\left(c_{\Delta} r_{\mathrm{scr}} / R_{\Delta}\right)}{F\left(c_{\Delta} r / R_{\Delta}\right)}\right) .
\end{aligned}
$$

We then form the same average via Eq. (27). As shown in Fig. 3 (thin black lines), this approximation predicts the onset of the chameleon screening quite well, though the predicted transition between unscreened and screened regimes is somewhat too sharp. Nevertheless, this simple model can be useful in interpolating the numerical results for different values of $f_{R 0}$.

\section{DGP}

In the DGP braneworld scenario [16], matter and radiation live on a four-dimensional brane in five-dimensional Minkowski space. The action is constructed so that on scales larger than the crossover scale $r_{c}$, gravity is five dimensional, while it becomes four dimensional on scales smaller than $r_{c}$. This model admits a homogeneous cosmological solution on the brane, which obeys a modifed Friedmann equation [40]:

$$
H^{2} \pm \frac{H}{r_{c}}=8 \pi G\left[\bar{\rho}+\rho_{\mathrm{DE}}\right] .
$$

The sign on the lhs is determined by the choice of embedding of the brane. The negative sign is called the selfaccelerating branch, since it allows for accelerated expansion even in the absence of a cosmological constant. The positive sign is called the normal branch, which does not exhibit self-acceleration. Here, we consider models of both branches (see [18,20,41]): a self-accelerating model without a $\Lambda$ term $\left(\rho_{\mathrm{DE}}=0\right)$, sDGP, where $r_{c} \sim 6000 \mathrm{Mpc}$ is adjusted to best match $\mathrm{CMB}$ and expansion history constraints [42] (note that this model is in $\sim 4-5 \sigma$ conflict with current data); and normal-branch models with a dark energy component $\rho_{\mathrm{DE}}$ adjusted so that the expansion history is exactly $\Lambda \mathrm{CDM}$ [20]. In that case, $r_{c}$ is a free parameter, and we chose values of $500 \mathrm{Mpc}$ (nDGP-1) and $3000 \mathrm{Mpc}$ (nDGP-2). The remaining cosmological parameters are summarized in Table I. For both sDGP and nDGP models, we have also performed ordinary GR simulations employing the same expansion history and initial conditions as for the DGP simulations.

On subhorizon scales, and scales smaller than the crossover scale $r_{c}$, DGP braneworld models can be accurately described as scalar-tensor theory [43], where the branebending mode $\varphi$ mediates an additional attractive (normal branch) or repulsive (self-accelerating branch) force. Gravitational forces in DGP are governed by

$$
\nabla \Psi=\nabla \Psi_{N}+\frac{1}{2} \nabla \varphi
$$

The $\varphi$ field is sourced by matter overdensities similar to the usual GR potentials, but has quadratic self-interactions that suppress the field once density contrasts become nonlinear. The full equation for the $\varphi$ field is (assuming $a=1$; see e.g. [44])

$$
\nabla^{2} \varphi+\frac{r_{c}^{2}}{3 \beta}\left[\left(\nabla^{2} \varphi\right)^{2}-\left(\nabla_{i} \nabla_{j} \varphi\right)\left(\nabla^{i} \nabla^{j} \varphi\right)\right]=\frac{8 \pi G}{3 \beta} \delta \rho .
$$

Here $\beta$ is determined by the expansion rate $H(a)$ via

$$
\beta(a)=1 \pm 2 H(a) r_{c}\left(1+\frac{\dot{H}(a)}{3 H^{2}(a)}\right),
$$

where the positive (negative) sign is valid for the normal (self-accelerating) branch. The present-day values for $\beta$ are given in Table I.

While analytical solutions to Eq. (45) do not exist in the general case, the case of a spherically symmetric mass is solvable in terms of closed expressions $[44,45]$. In particu- 
lar, one obtains the following equation for the gradient of $\varphi$ [41]:

$$
\begin{gathered}
\frac{d \varphi}{d r}=\frac{G \delta M(<r)}{r^{2}} \frac{4}{3 \beta} g\left(\frac{r}{r_{*}(r)}\right), \\
g(\xi)=\xi^{3}\left(\sqrt{1+\xi^{-3}}-1\right) .
\end{gathered}
$$

$r_{*}(r)$ in Eq. (47) is the $r$-dependent Vainshtein radius defined as

$$
r_{*}(r)=\left(\frac{16 G \delta M(<r) r_{c}^{2}}{9 \beta^{2}}\right)^{1 / 3} .
$$

Note that $r / r_{*}$ is a function of the average overdensity $\delta \rho(<r)$ within $r$. Specifically, scaling to a halo with mass $M_{\Delta}$ and radius $R_{\Delta}$ determined by a fixed overdensity $\Delta$ and neglecting the small difference between $M$ and $\delta M$, we have

$$
\frac{r}{r_{*}(r)}=(\varepsilon \Delta)^{-1 / 3} x\left(\frac{M(<x)}{M_{\Delta}}\right)^{-1 / 3},
$$

where $x=r / R_{\Delta}$ and the quantity $\varepsilon$ is determined by the background cosmology:

$$
\varepsilon=\frac{8}{9 \beta^{2}}\left(H_{0} r_{c}\right)^{2} \Omega_{m} a^{-3} .
$$

At $a=1, \varepsilon=0.32$ for sDGP, and $0.002 / 0.023$ for nDGP-1/nDGP-2, respectively. Using Eq. (3), (44), and (47), we then have

$$
\mathfrak{g}_{\mathrm{DGP}}(r)=1+\frac{2}{3 \beta} g\left(\frac{r}{r_{*}(r)}\right) .
$$

On large scales where $\delta \rho(<r) \ll \bar{\rho}, r$ is much larger than $r_{*}$ so that $g(\xi) \rightarrow 1 / 2$ and $d \varphi / d r$ becomes simply proportional to $d \Psi_{N} / d r$. In this limit, $\mathfrak{g}_{\mathrm{DGP}}=\mathfrak{g}_{\mathrm{DGP}, \text { lin }}=$ $1+1 /(3 \beta)$. This is the same expression one would obtain by simply neglecting the nonlinear terms in Eq. (45). On small scales where $r \ll r_{*}$, modified forces are suppressed by $(\varepsilon \bar{\delta})^{-1 / 2}$, where $\bar{\delta}=\delta \rho(<r) / \bar{\rho}$ is the average overdensity within $r$.

Note that the specific tensorial structure of the nonlinearities in Eq. (45) is crucial to recover the linearized expression $\mathfrak{g}_{\mathrm{DGP}, \mathrm{lin}}$. It is possible to simplify Eq. (45) by neglecting the tensorial structure, resulting in a Poisson equation for $\varphi$ with a source term given by a nonlinear function of $\delta \rho$ [21]. However, this simplification qualitatively changes the large-distance behavior of the Vainshtein mechanism $[21,41]$. Thus, it will turn out to be crucial that the simulations solve the full Eq. (45) for our comparison with the theoretical predictions from Eqs. (47) and (52).

Note that in the Vainshtein limit,

$$
\varphi\left(r \ll r_{*}\right) \approx C \frac{G \delta M(<r)}{r_{*}} \propto \Psi_{N}(r) \frac{r_{*}}{r},
$$

where $C$ is a constant of order unity [41]. Hence, the $\varphi$ field itself is suppressed less than the modified forces by a factor of $\left(r / r_{*}\right)^{1 / 2}$. However, only the forces are observable. This shows that in theories with nonlinear interactions such as DGP, quantifying departures from GR in terms of forces are more appropriate than the parameter $\gamma_{\mathrm{PPN}}$ defined in terms of the potentials [Eq. (5)].

For a mass profile with constant density ("tophat"), the force enhancement in Eq. (52) is independent of radius; for more general profiles, however, this is not the case (see also [41] for a detailed discussion). Figure 4 shows the relative force enhancement $g(r)$ as a function of radius $r / R_{\Delta}$ in the case of an NFW halo, for the different DGP models (see $[18,20]$, Table I). We also show the ( $r$-independent) linearized value $\mathfrak{g}_{\mathrm{DGP}, \text { lin }}$ for the nDGP models, which is recovered only at very large scales when the average density becomes $\lesssim \varepsilon^{-1}$ (for sDGP, $\mathfrak{g}_{\mathrm{DGP}, \text { lin }} \approx 0.76$ is beyond the range of the plot).

Since $\mathfrak{g}_{\text {DGP }}$ depends on only the average overdensity $\delta \rho / \bar{\rho}$, which is completely determined by $\Delta$ and $c_{\Delta}$, the force enhancement does not directly depend on the halo mass. Also, it is insensitive to the large-scale environment of the halo. These are two crucial distinctions from the $f(R)$ case.

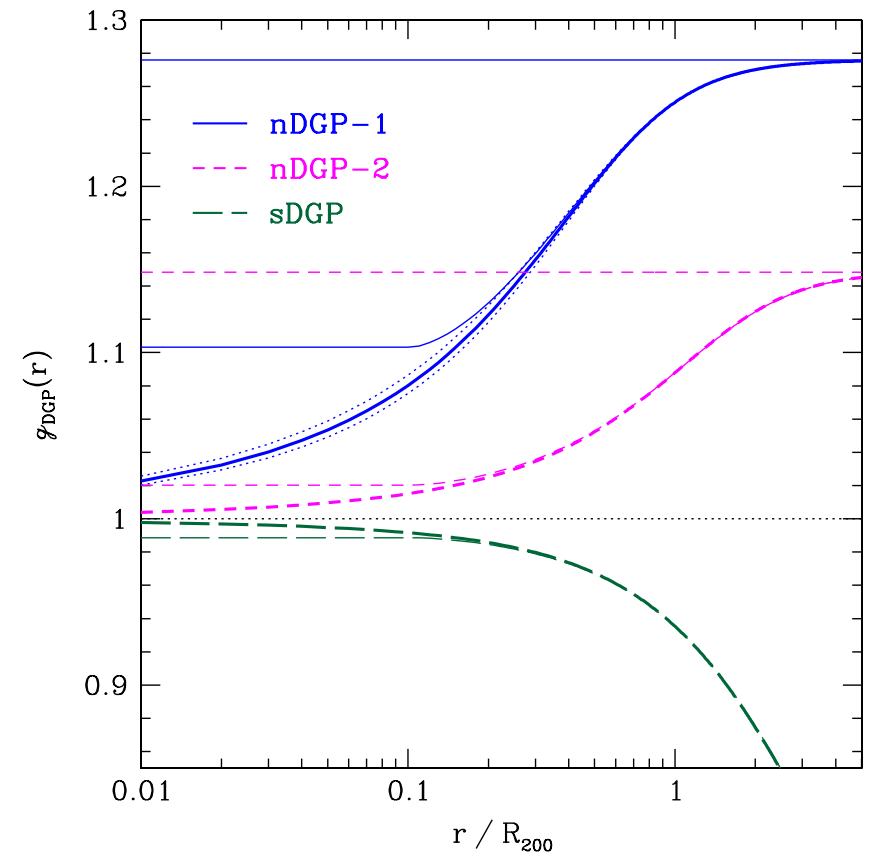

FIG. 4 (color online). $\mathfrak{g}_{\mathrm{DGP}}(r)$ [Eq. (52)] as a function of the scaled radius $r / R_{\Delta}$ for an NFW halo, for the DGP models. The thin horizontal lines show the linearized expression $\mathfrak{g}_{\mathrm{DGP}, \mathrm{lin}}=$ $1+1 /(3 \beta)$, while the thin lines deviating at small $r$ show the results when using a capped density profile with $r_{\text {cap }}=$ $0.125 \mathrm{Mpc} / h$ (see Sec. III B). In all cases, we assumed $\Delta=$ 200 and a concentration of $c_{\Delta}=5$. For nDGP-1, we also show the effect of varying the concentration by $\pm 20 \%$ (dotted lines). Note that $\mathfrak{g}_{\mathrm{DGP}}$ is independent of the halo mass. 


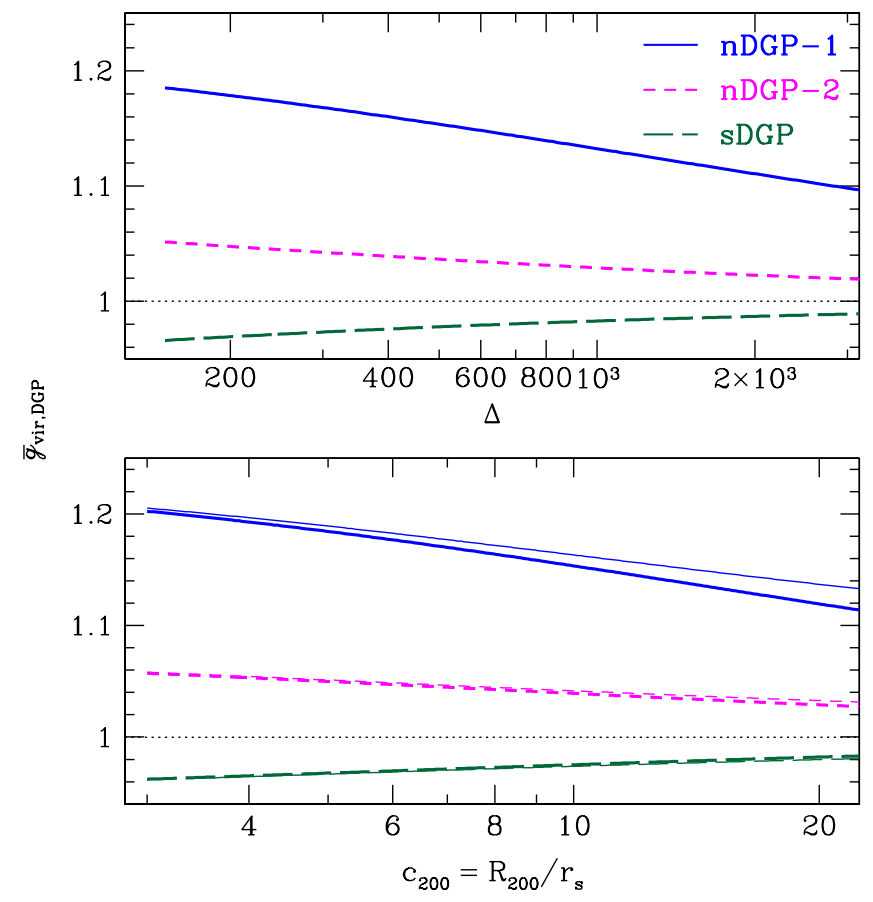

FIG. 5 (color online). Averaged force deviation $\overline{\mathrm{g}}_{\text {vir }}$ [Eq. (27)] for DGP models as a function of the overdensity $\Delta$ (top panel) and the halo concentration $c_{200}$ for an NFW halo (bottom panel). For the top panel we have scaled the concentration with $R_{\Delta}$ to keep $r_{s}$ fixed (corresponding to $c_{200} \approx 5$.8). The thin lines in the bottom panel show the results for a density profile capped at $r_{\text {cap }}=0.125 \mathrm{Mpc} / h$ for comparison with simulation results (assuming $M_{200}=10^{14} M_{\odot} / h$; see Sec. III B).

Figure 5 shows $\overline{\mathrm{g}}_{\mathrm{vir}, \mathrm{DGP}}$ defined in Eq. (27) as a function of the overdensity $\Delta$ (keeping $r_{s}$ fixed at a value expected for a $10^{14} M_{\odot} / h$ halo), and the halo concentration. Clearly, $\overline{\mathfrak{g}}_{\text {vir,DGP }}$ does depend somewhat on the halo profile and the overdensity criterion chosen. The general trend is that more concentrated halos lead to a stronger suppression of the modified forces, since they have higher average density at small radii. The same holds when increasing $\Delta$. The dependence on $c$ and $\Delta$ is strongest for nDGP-1, which also shows the strongest evolution of $g_{\mathrm{DGP}}(r)$. The dependence on the density profile has to be taken into account when comparing with simulation results (Sec. III B), as well as for the comparison with observations that measure the dynamical mass within different $R_{\Delta}$ (Sec. IV).

\section{COMPARISON WITH SIMULATIONS}

In order to benchmark our theoretical expectations, we will now compare them to the results of the self-consistent $N$-body simulations of $f(R)$ gravity presented in $[17,39]$ and of DGP $[18,20]$. For each model, we have simulated several box sizes. The number of runs for each model and box size, as well as the grid resolution, are summarized in Table II. Halos are identified using a spherical overdensity halo finder as described in $[32,41]$. The halo finder returns
TABLE II. Number of runs for each box size and minimum mass cuts for $\sigma_{v}^{2}$ and $\overline{\mathfrak{g}}_{\text {vir }}$ measurement.

\begin{tabular}{lccccc}
\hline \hline & & \multicolumn{4}{c}{$L_{\text {box }}\left(h^{-1} \mathrm{Mpc}\right)$} \\
& Model & 400 & 256 & 128 & 64 \\
\hline No. of runs & $f(R)^{\mathrm{a}}$ & 6 & 6 & 6 & 6 \\
& $\mathrm{sDGP}$ & 6 & 6 & 6 & 6 \\
& $\mathrm{nDGP}$ & 3 & 3 & 3 & 6 \\
$M_{\mathrm{h}, \min }\left(10^{14} M_{\odot} / h\right)$ & & 63.5 & 16.7 & 2.08 & 0.26 \\
$r_{\text {cell }}\left(h^{-1} \mathrm{Mpc}\right)$ & & 0.78 & 0.50 & 0.25 & 0.125 \\
\hline \hline
\end{tabular}

${ }^{\mathrm{a}}$ For each value of $\left|f_{R 0}\right|$.

${ }^{\mathrm{b}}$ For nDGP-1 and nDGP-2 each.

the center-of-mass position as well as mass $M_{\Delta}$ of the halo as determined from the particles within $R_{\Delta}$, such that $M_{\Delta} /\left(4 \pi / 3 R_{\Delta}^{3}\right)=\bar{\rho} \Delta$. Our choice of $\Delta$ is the one adopted in $[32,41]: \Delta=300$ for the $f(R)$ simulations, and $\Delta=200$ in the DGP case. Our particle-mesh simulations are of limited resolution, and we can only use the best-resolved halos for our study, i.e. massive halos in the two smallest boxes. This limits our statistical sample of halos.

First, in order to measure $\mathrm{g}(r)$ as a function of the radius, we select the most well-resolved halos whose radii $R_{\Delta}$ are at least 10 grid cells, which is only satisfied for halos in our smallest box, $L_{\text {box }}=64 \mathrm{Mpc} / h$. For this box, this corresponds to a minimum mass of $\sim 1.6 \times 10^{14} M_{\odot} / h$, which depending on the model results in a very small sample of 2-40 halos. For each halo, we then measure contributions to the potential energy $W(r)$ in spherical shells around the center-of-mass via

$$
W(r)=\frac{1}{N} \sum_{\left|r_{i}-r\right| \leq \Delta r}\left(\mathbf{x}_{i}-\mathbf{x}_{h}\right) \cdot \nabla \Psi\left(\mathbf{x}_{i}\right),
$$

where the sum runs over particles whose distance $r_{i}=$ $\left|\mathbf{x}_{i}-\mathbf{x}_{h}\right|$ from the center of mass of the halo is within the radial bin, and $N$ is the number of contributing particles. The derivative of the potential is evaluated at the position of each particle in the same way as it is done in the particle propagation of the $N$-body simulation (bilinear interpolation). In addition to $W(r)$ derived from the dynamical potential $\Psi$, we also measure the corresponding Newtonian quantity $W_{N}(r)$, where the Newtonian potential $\Psi_{N}$ is determined from Eq. (14) using the same density field. The ratio of the two is our estimated force enhancement:

$$
\mathfrak{g}_{\text {meas }}(r)=\frac{W(r)}{W_{N}(r)} .
$$

To some extent, resolution effects can be expected to cancel out in Eq. (55). Below we will show profiles down to $r=r_{\text {cell }}$, though one should keep in mind that the $g$ profiles cannot be considered reliable below $r \sim 4 r_{\text {cell }}$.

Because of the resolution requirements and small sample size, we cannot study any evolution with mass in the 
$g(r)$ profiles. The stringent resolution requirements can be relaxed somewhat if we measure only an average force enhancement, for example, $\overline{\mathfrak{g}}_{\text {vir }}$. Assuming a scaling following the virial theorem, we can either measure an average of $\mathbf{x} \cdot \nabla \Psi$, related to the trace of the potential energy tensor $W$ [Eq. (7)], or we can measure the velocity dispersion, related to the kinetic energy given by Eq. (9). The first approach has the advantage that we can measure $\overline{\mathrm{g}}_{\text {vir }}$ on a halo-by-halo basis, by calculating $W$ using both the modified potential $\Psi$ and the Newtonian potential $\Psi_{N}$ [similar to what was done for $\overline{\mathfrak{g}}_{\text {meas }}(r)$ ]. The estimator of $\overline{\mathfrak{g}}_{\text {vir }}$ for a given halo is then defined by

$$
\overline{\mathfrak{g}}_{\text {vir,meas }}=\frac{\sum_{r_{i}<R_{\Delta}}\left(\mathbf{x}_{i}-\mathbf{x}_{h}\right) \cdot \nabla \Psi\left(\mathbf{x}_{i}\right)}{\sum_{r_{i}<R_{\Delta}}\left(\mathbf{x}_{i}-\mathbf{x}_{h}\right) \cdot \nabla \Psi_{N}\left(\mathbf{x}_{i}\right)},
$$

where the sum runs over particles within the halo radius $R_{\Delta}$. Note that the sum over particles automatically results in a density weighting of g. Again, we expect that in this ratio resolution issues cancel to a certain extent. Some effects of the finite resolution will become apparent when comparing with the theoretical predictions below.

The second approach, measuring the halo velocity dispersions, is also interesting since it gives an independent estimate of the modified forces. Specifically, we define the (one-dimensional) velocity dispersion of particles in a halo as follows:

$$
\begin{gathered}
\sigma_{v}^{2}=\frac{1}{3 N_{p}} \sum_{\left|\mathbf{x}-\mathbf{x}_{h}\right|<R_{\Delta}}\left(\mathbf{v}_{i}-\mathbf{v}_{h}\right)^{2}, \\
\mathbf{v}_{h}=\frac{1}{N_{p}} \sum_{\left|\mathbf{x}-\mathbf{x}_{h}\right|<R_{\Delta}} \mathbf{v}_{i},
\end{gathered}
$$

where the sum runs over particles within the halo radius $R_{\Delta}, N_{p}$ is the number of those particles, and $\mathbf{v}_{i}-\mathbf{v}_{h}$ denotes the velocity of the particle with respect to the center of mass of the halo. Note that in our normalization of $\sigma_{v}$, the kinetic energy Eq. (9) is given by $T=$ $3 / 2 M_{\Delta} \sigma_{v}^{2}$. From the results of Sec. II A, we expect that when averaged over many halos,

$$
\frac{\sigma_{v, \mathrm{MG}}^{2}}{\sigma_{v, \mathrm{GR}}^{2}}=\overline{\mathrm{g}}_{\mathrm{vir}}
$$

where $\sigma_{v, \mathrm{MG}}^{2}$ is the velocity dispersion measured in the modified gravity simulations, while $\sigma_{v, \mathrm{GR}}^{2}$ is measured in the corresponding GR simulations. Note that in this measurement, we can only compare the average of many halos in the modified gravity simulations to that in GR, rather than calculating g on a halo-by-halo basis. Hence, Eq. (59) results in a noisier measurement of $\overline{\mathfrak{g}}_{\text {vir }}$ than Eq. (56). However, the particle velocity dispersion, which has gone through the relaxation and virialization process, is much more closely related to observables than the averaged gravitational force strength Eq. (56), which can never be measured directly in reality. Thus, it is worthwhile to crosscheck our results obtained from Eq. (56) with the halo velocity dispersions.

In order to determine for which halos we can reliably measure Eqs. (56) and (57), we calculate the velocity dispersion of halos in the standard GR simulations and compare it to the expected virial scaling. Figure 6 shows the measured velocity dispersion scaled as $\sigma_{v}^{2} / M_{\Delta}^{2 / 3}$, as a function of the halo radius $R_{300}$ in grid cells, for the different simulation boxes. Since the virial theorem has been found to hold in simulated halos [31], $\sigma_{v}^{2} / M_{\Delta}^{2 / 3}$ should be independent of the halo mass (Sec. II). We see that, within the significant scatter, this is approximately true for halos that are sufficiently resolved. We thus place a radius cut of $R_{\Delta} \geq 5.4$ grid cells. Since $M_{\Delta}=(4 \pi / 3) \Delta \bar{\rho} R_{\Delta}^{3}$, this corresponds to a fixed mass cut for a given simulation box size, which is listed in Table I. Fortunately, the statistics are then sufficient to study $\overline{\mathfrak{g}}_{\text {vir }}$ and $\sigma_{v}^{2}$ as functions of mass.

\section{A. $f(\boldsymbol{R})$}

We begin with the measurement of $\mathfrak{g}_{f(R)}$ for the wellresolved halos. Figure 7 shows the simulation measurements and predictions of the spherical relaxation code, for the strong field $\left(\left|f_{R 0}\right|=10^{-4}\right)$ and the weak field $\left(10^{-6}\right)$. As expected from Fig. 3, the halos in this mass range $\left(M_{300} \approx 1.6-7 \times 10^{14} M_{\odot} / h\right)$ are unscreened in the strong

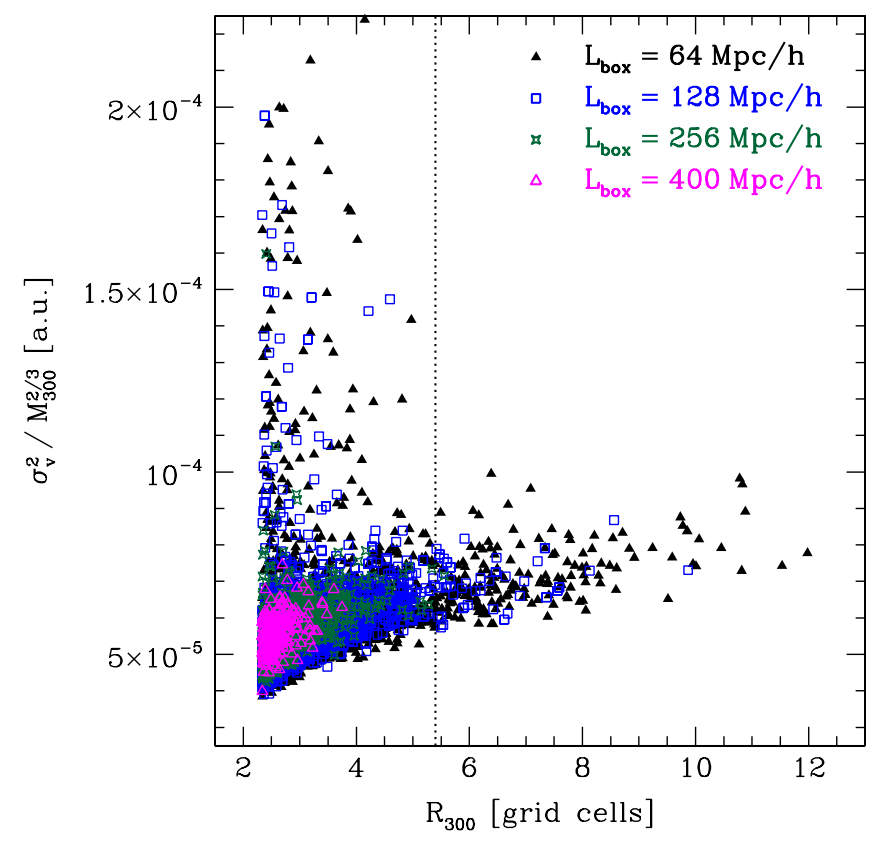

FIG. 6 (color online). Velocity dispersion $\sigma_{v}$ scaled to the virial expectation $\left(\sigma_{v}^{2} \propto M^{2 / 3}\right)$, measured for halos in the GR simulations, as a function of the halo radius in grid cells. Velocity dispersions are only reliably measured for the most well-resolved halos with $R_{300} \geq 5.4$ grid cells (indicated by the vertical line). 
DYNAMICAL MASSES IN MODIFIED GRAVITY

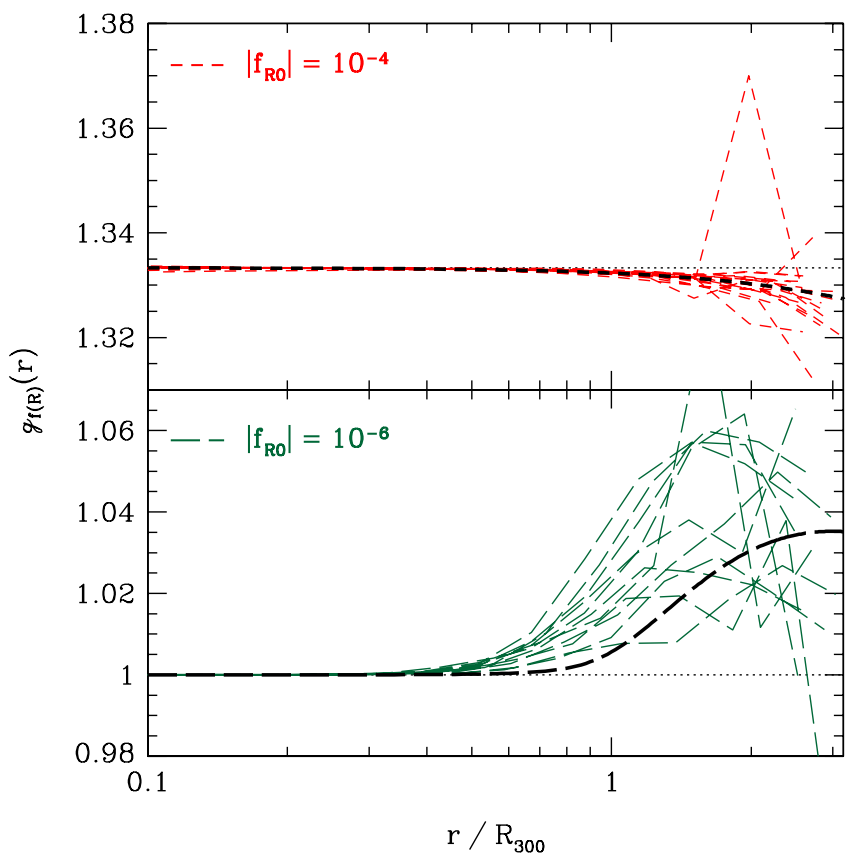

FIG. 7 (color online). $\quad \mathfrak{g}_{f(R)}(r)$ measured using Eqs. (54) and (55) (thin lines), for the most well-resolved halos $\left(R_{300}>10\right.$ grid cells) in the $f(R)$ simulations, for the strong field $\left|f_{R 0}\right|=10^{-4}$ and weak field $\left|f_{R 0}\right|=10^{-6}$. The mass range of the halos shown here is $M_{300}=1.6-7 \times 10^{14} M_{\odot} / h$. The thick lines show the results of the relaxation code (for $M_{300}=3 \times 10^{14} M_{\odot} / h$ ).

field simulations, $\mathfrak{g}_{f(R)}=4 / 3$, and screened in the weak field, $\mathfrak{g}_{f(R)} \rightarrow 1$. In the latter case, there is a regime around 1-3 $R_{300}$ where the screening is not complete. At larger distances, the Yukawa suppression again becomes noticeable $\left(\lambda_{C} \approx 3 \mathrm{Mpc}\right.$ for $\left.\left|f_{R 0}\right|=10^{-6}\right)$. The numerical results for the spherically symmetric case match the overall behavior well for both field values, although there is a hint that it slightly overestimates the screening in the weak field case.

The results for the intermediate field value $\left|f_{R 0}\right|=10^{-5}$ are shown in Fig. 8; this case is most interesting since the few $\times 10^{14} M_{\odot} / h$ halos are in the transition region between the screened and unscreened limits (Fig. 3). Hence we have split the halo sample into a lower mass sample around $2 \times$ $10^{14} M_{\odot} / h$ and a high mass sample with two halos around $7 \times 10^{14} M_{\odot} / h$. Clearly, the scatter in the modified force profiles is significant. Nevertheless, the stronger screening effect in the higher mass halos is noticeable. The spherical relaxation results (Sec. II C), which were calculated separately for the mean halo mass of each sample, match the full simulation profiles remarkably well. At small radii, the transition to the fully screened values is apparently too steep. A possible explanation for this is that the halos in the $\mathrm{N}$-body simulations are not truly spherical, but in general triaxial. A triaxial halo will have a somewhat shallower potential well, reducing the chameleon screening effect. Furthermore, the screening will happen at different radii
PHYSICAL REVIEW D 81, 103002 (2010)

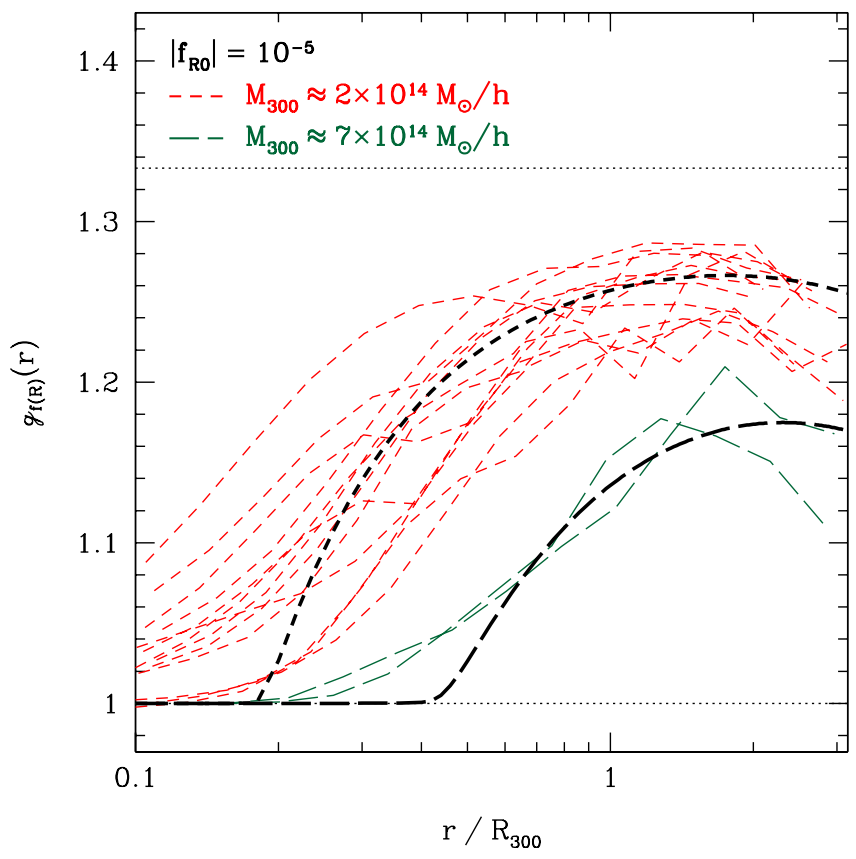

FIG. 8 (color online). Same as Fig. 7, but for the intermediate field value $\left|f_{R 0}\right|=10^{-5}$. We have separated the halo sample into lower mass halos with $M_{300}=1.6-2.5 \times 10^{14} M_{\odot} / h$ and two higher mass halos with $M_{300}=6-7 \times 10^{14} M_{\odot} / h$.

along the different axes, so that a potentially sharp transition in the spherical case is washed out over a certain radius range. In addition, the innermost Newtonian potential well is not as deep in the simulations as predicted for a perfect NFW halo due to the finite resolution. We also reiterate that the profiles only become reliable at $r \sim$ $(0.3-0.4) R_{300}$ for these halos.

Next, we look at $\bar{g}_{\text {virmeas }}$ in the larger sample of halos. Figure 9 shows the results for the three field values and the predictions of $\overline{\mathrm{g}}_{\mathrm{vir}, f(R)}(M)$ from the spherical relaxation code. We again see a very good match for all field values and over the entire mass range probed by this halo sample, $3 \times 10^{13} M_{\odot} / h<M_{300}<3 \times 10^{15} M_{\odot} / h$. For the intermediate field value, which again shows the most interesting behavior in this mass range, we see that the screening effect is slightly overpredicted in the spherically symmetric approximation. Again, this could be due to halo triaxiality and to resolution effects that reduce the value of $\Psi_{N}$ in the inner parts of the halo.

For the intermediate field, some outliers are seen in Fig. 9. These halos, especially around $3-8 \times 10^{13} M_{\odot} / h$, show a stronger screening of the modified forces than the large majority of halos at that mass, and stronger than predicted for isolated spherical NFW halos. This would seem consistent with halos being screened by a larger scale potential well in which they are situated. To test this hypothesis, we have selected halos in the intermediate field simulations, which have a more massive neighboring halo in their immediate vicinity. More precisely, we ask that 
FABIAN SCHMIDT

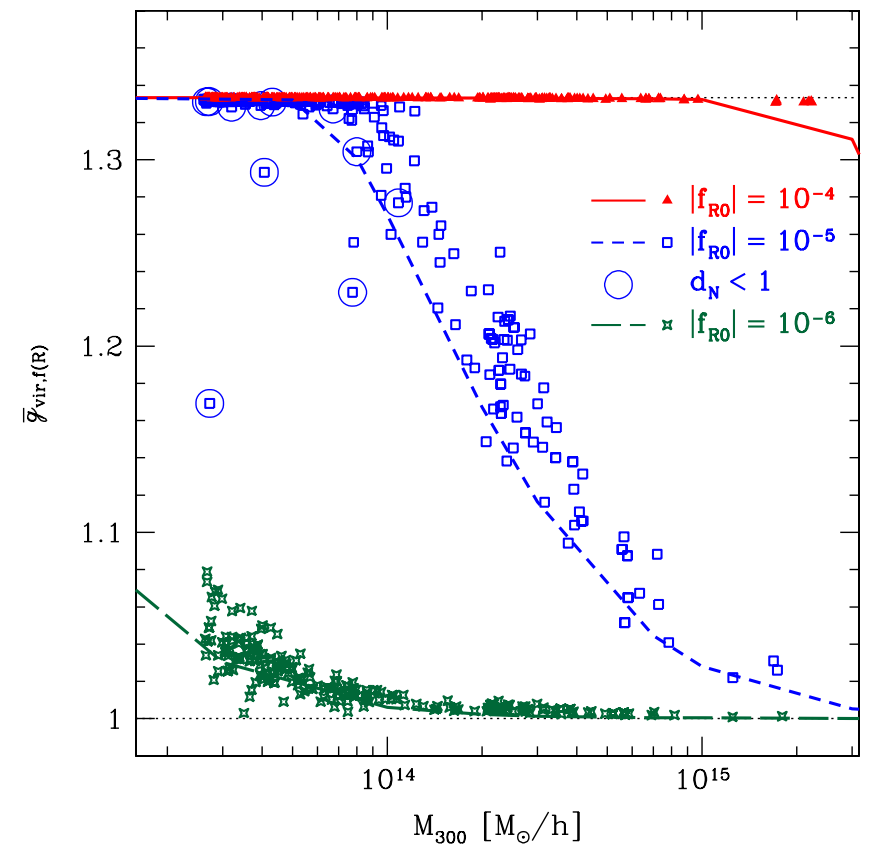

FIG. 9 (color online). $\quad \overline{\mathfrak{g}}_{\text {vir }}$ measured via Eq. (56) for wellresolved halos $\left(R_{300}>5.4\right.$ grid cells $)$ in the $f(R)$ simulations (points). The results confirm the theoretical predictions from Sec. IIC, shown as lines (spherical relaxation results from Fig. 3). The circled points are halos that have a more massive halo in their immediate vicinity (see text).

$$
d_{N} \equiv \frac{\left|\mathbf{x}_{N}-\mathbf{x}_{h}\right|}{R_{\Delta, N}+R_{\Delta, h}}<1,
$$

where $h$ denotes the halo itself and $N$ denotes the closest neighboring halo ${ }^{2}$ with $M_{\Delta, N}>M_{\Delta, h}$. These halos make up less than $5 \%$ of the whole sample and are circled in Fig. 9. In fact, three of the outliers have a close massive neighbor, which is strong evidence for the hypothesis of environmental effects as cause for the enhanced screening (the fourth most obvious outlier has $d_{N} \approx 1.2$ ).

Finally, we can look at the effect of the modified forces on the particle velocity dispersion of halos, a noisier measurement but one that probes the effect after the reprocessing through gravitational collapse and virialization. Figure 10 shows the scaled velocity dispersion $\sigma_{v}^{2} / M^{2 / 3}$, for the same halos as in Fig. 9 and scaled to values expected from Sec. II, as a function of mass. We show the results for GR simulations as well as the weak $\left(\left|f_{R 0}\right|=10^{-6}\right)$ and strong field $\left(\left|f_{R 0}\right|=10^{-4}\right) f(R)$ cases. After fitting a constant to the GR simulations, we multiply the theoretical predictions by this constant. Albeit noisy, the results of the halo-by-halo measurement of $\overline{\mathrm{g}}_{\mathrm{vir}, f(R)}$ are confirmed: for

\footnotetext{
${ }^{2}$ This criterion formally says that the halos are overlapping. Such an overlap is unavoidable when defining halos via spherical overdensities. In our halo finding algorithm, the particles in the overlap region are not double counted, but counted toward the more massive halo.
}

PHYSICAL REVIEW D 81, 103002 (2010)

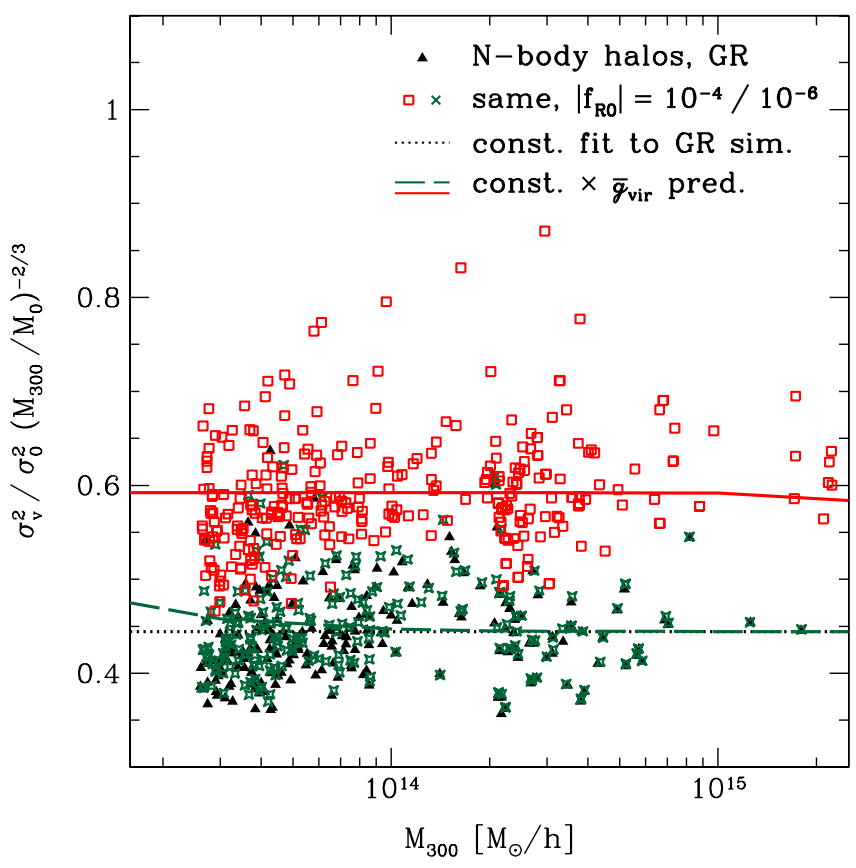

FIG. 10 (color online). Scaled velocity dispersion $\sigma_{v}^{2} / M^{2 / 3}$ measured in GR and $f(R)$ simulations. The measurements were scaled to values expected for NFW halos (Sec. II): $\sigma_{0}^{2}=$ $1.79 \times 10^{-5} c^{2}, M_{0}=10^{15} M_{\odot} / h$. The dotted black line shows a constant fit to the GR results. Solid and dashed lines show the predictions of the model of Sec. II C scaled by the GR value.

the strong field, all halos are in the linearized field regime where forces, and hence $\sigma_{v}^{2}$, are enhanced by a factor of $4 / 3$. For the weak field, all halos except at the very lowest masses probed by the simulations are in the chameleon regime. In case of the intermediate field $\left(\left|f_{R 0}\right|=10^{-5}\right)$, Fig. 11 shows that the transition between screened and unscreened regimes at few $\times 10^{14} M_{\odot} / h$ is indeed seen in the halo velocity dispersions as well. These results confirm that the theoretical predictions for the modified gravitational force can in principle be probed by observable quantities such as velocity dispersions (see Sec. IV).

\section{B. DGP}

The force modifications in DGP are, to first order, independent of the halo mass and environment. However, they do depend on the detailed halo profile. Before comparing the predictions from Sec. II D with the simulation results, we have to take into account the effects of the finite resolution. While the NFW profile we used throughout Sec. II is a very good match to high-resolution simulations, in our fixed-grid simulations the density profile is, in fact, softened on scales of a grid cell. This softening of the density profile will affect $\mathfrak{g}_{\text {DGP }}(r)$ through the average overdensity within $r$. Thus, for comparison with the simulation results we assume a "capped" density profile instead of NFW all the way to $r=0$. More precisely, we cap the density profile at a constant value of $\rho_{\text {cap }}=\rho_{\mathrm{NFW}}\left(r_{\text {cap }}\right)$ 
DYNAMICAL MASSES IN MODIFIED GRAVITY

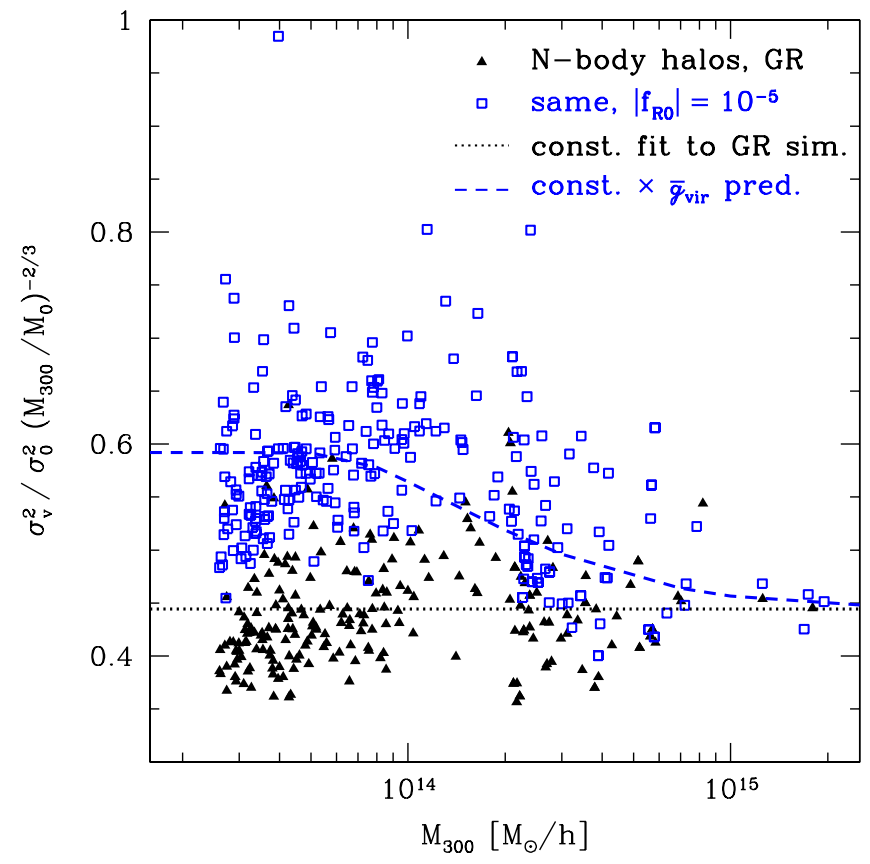

FIG. 11 (color online). Same as Fig. 10, but for the intermediate $f(R)$ field value $\left|f_{R 0}\right|=10^{-5}$.

for $r<r_{\text {cap }}{ }^{3}$ For the halos measured in the smallest box, a natural choice is $r_{\text {cap }}=r_{\text {cell }}=0.125 \mathrm{Mpc} / h$ (Table II). Figure 4 shows the effect of this softened density profile on $\mathfrak{g}_{\text {DGP }}(r)$. In particular, it increases the force modification since the inner density is suppressed, thus artificially weakening the Vainshtein mechanism.

Figure 12 shows the measured $\mathfrak{g}_{\mathrm{DGP}}(r)$ from the simulations, together with the predictions using the capped density profile. First, it is evident that the scatter in $g$ is much smaller in DGP than it is for $f(R)$, due to the locality of the Vainshtein mechanism. For all three models, the agreement of the simple spherically symmetric NFW model with the simulations is impressive. Note that we have not adjusted any parameters to match the simulation results; this measurement thus also constitutes a nontrivial test of the DGP simulations. At $r \sim R_{200}$, the theoretical prediction slightly underestimates the suppression of the force modification (by 1\%-3\%), which is presumably due to slight differences in the actual density profiles from the one assumed in the predictions (pure spherical NFW profile). The large scatter at $r \gtrsim 2 R_{200}$ is due to the effect of gravitationally unbound ambient matter in the environment of the halos, which dominates $\delta \rho$ at these distances. Note that, in particular, for nDGP-1, the Vainshtein mechanism does not completely suppress the force modifications within halos even on scales as small as $\sim 100 \mathrm{kpc}$.

\footnotetext{
${ }^{3}$ Note that the halo radius for a given mass is slightly increased when using the capped density profile, in order to match the fixed overdensity $\Delta=200$.
}

PHYSICAL REVIEW D 81, 103002 (2010)

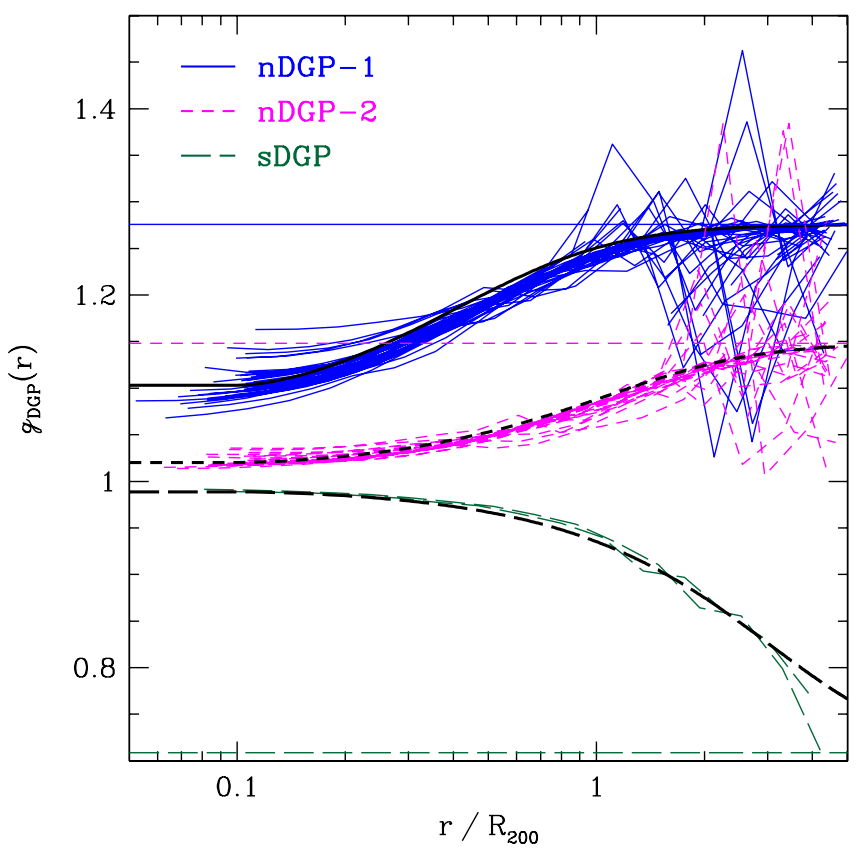

FIG. 12 (color online). $\mathfrak{g}_{\text {DGP }}(r)$ measured using Eqs. (54) and (55) for the most well-resolved halos $\left(R_{200}>10\right.$ grid cells) in the DGP simulations (thin lines). The thick lines show the prediction of Eq. (52), using a capped NFW profile with $r_{\text {cap }}=$ $0.125 \mathrm{Mpc} / h$ (see text). The thin horizontal lines show $\mathfrak{g}_{\mathrm{DGP}, \text { lin }}$ for each model. The halos shown here have masses $M_{200}=$ $1.6-7 \times 10^{14} M_{\odot} / h$.

We now turn to $\overline{\mathfrak{g}}_{\text {vir }}$ as measured from Eq. (56) in the halo sample with $R_{200} \geq 5.4$ grid cells. Figure 13 shows the measurements for the three DGP models. As expected, $\overline{\mathrm{g}}_{\text {vir,DGP }}$ is approximately constant as a function of mass. The model predictions from Sec. IID are shown as gray bands. Here, we have used the concentration relation Eq. (26) (more precisely, $c=\max \{4, c(M)\}$ ), and the width of the band reflects a $\pm 20 \%$ spread in concentration. We again assumed a capped NFW profile with $r_{\text {cap }}=$ $0.125 \mathrm{Mpc} / h$, the effects of which are noticeable as a slight increasing trend of $\overline{\mathrm{g}}_{\mathrm{vir}}(M)$ in going toward the low-mass end for nDGP-1. Note that here we have included halos from different simulation box sizes $L_{\text {box }}=$ 64-256 Mpc/h, though the majority comes from the smallest box. Hence, one might wonder whether different values of $r_{\text {cap }}$ are required for different box sizes. However, within the limited statistics the measurements of $\overline{\mathfrak{g}}_{\text {vir }}$ from halos in different box sizes are in agreement. Hence, the data do not seem to require such a correction. We conclude that, within the uncertainties due to the halo density profiles, the measured values of $\overline{\mathfrak{g}}_{\text {vir,DGP }}$ are entirely consistent with the predictions. Furthermore, the scatter in the measured $\overline{\mathfrak{g}}_{\text {vir }}$ appears consistent with that expected for intrinsic variations of halo density profiles $(\Delta c / c \sim 0.2)$.

The results for $\overline{\mathfrak{g}}_{\mathrm{vir}, \mathrm{DGP}}$ are confirmed by the particle velocity dispersions of halos. Figure 14 shows the scaled velocity dispersion $\sigma_{v}^{2} / M^{2 / 3}$ in nDGP-1 and the corre- 


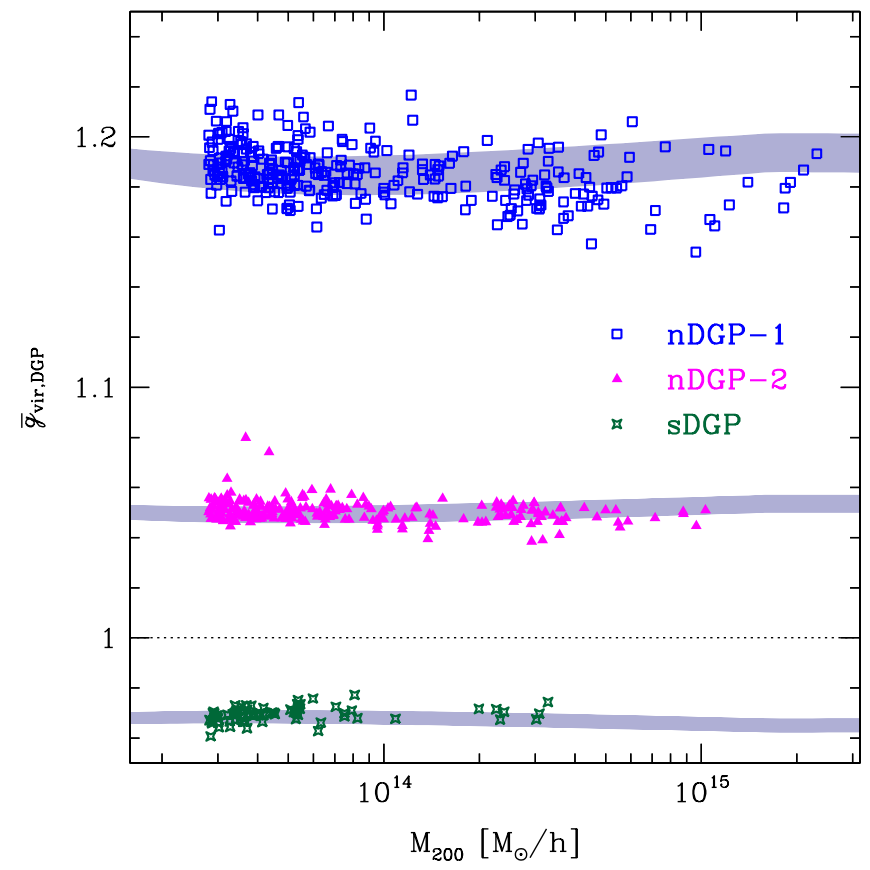

FIG. 13 (color online). $\quad \overline{\mathfrak{g}}_{\text {vir,DGP }}$ measured using Eq. (56) for well-resolved halos $\left(R_{200}>5.4\right.$ grid cells $)$ in the DGP simulations (points). The shaded bands show the model predictions from Sec. IID with a variation in the halo concentration by $\pm 20 \%$. We assumed capped NFW profiles with $r_{\text {cap }}=$ $0.125 \mathrm{Mpc} / h$.

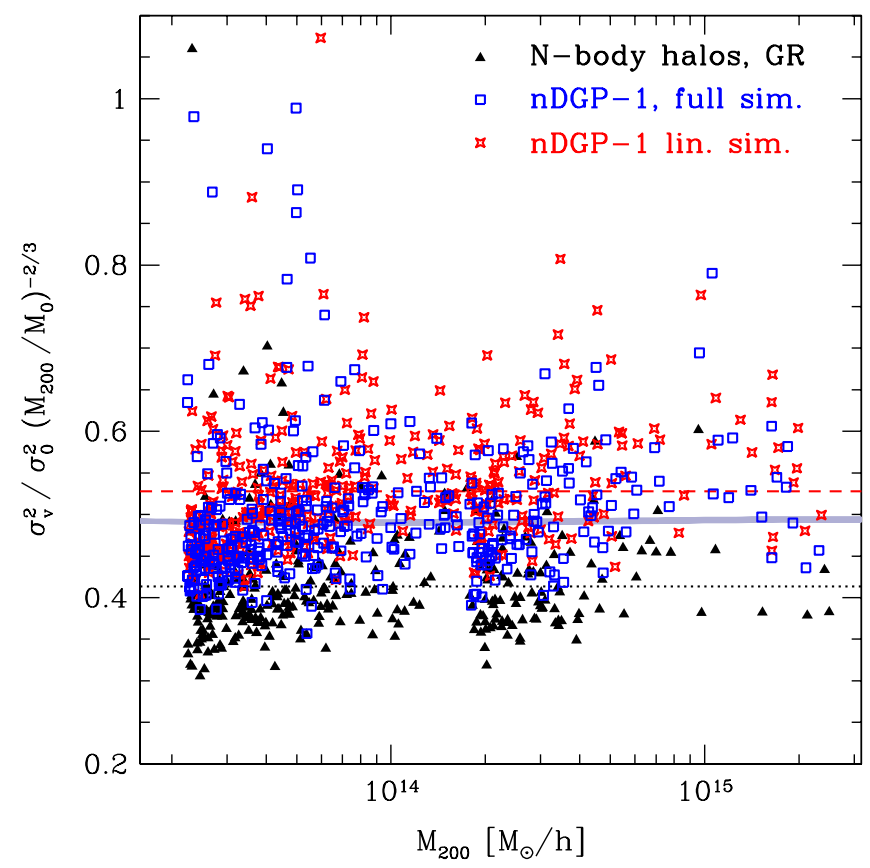

FIG. 14 (color online). Scaled velocity dispersion $\sigma_{v}^{2} / M^{2 / 3}$ measured in GR and nDGP-1 simulations $\left(\sigma_{0}\right.$ and $M_{0}$ are as defined in Fig. 10). The black dotted line shows the average value measured for the GR simulations. The red line shows this value multiplied by $\overline{\mathfrak{g}}_{\text {vir,DGPlin }}=1+1 /(3 \beta)$. The shaded band shows the corresponding prediction for $\overline{\mathfrak{g}}_{\text {vir,DGP }}$ from Fig. 13. sponding GR simulations. For comparison, we also show the result for the linearized DGP simulations, which use the scale-independent (but redshift-dependent) force enhancement obtained when linearizing the DGP equations $[18,20]$. Within the significant scatter in the $\sigma_{v}$ measurement, we found no significant evolution of the force enhancement with mass, as expected given the small trends with mass in Fig. 13.

In order to quantitatively compare the simulation results with model predictions, we determined the mean of $\sigma_{v}^{2} / M^{2 / 3}$ for each simulation type. In each case, the error on this mean is obtained by dividing the rms scatter by $\sqrt{N_{\text {halos }}}$. The measured ratio of the scaled velocity dispersion in the DGP simulations to that in the GR simulations is found to be

$$
\overline{\mathfrak{g}}_{\text {vir }}(\text { full DGP, meas })=1.212 \pm 0.014 .
$$

This is indeed close to the range of the theoretical predictions (for a capped NFW profile), 1.18-1.2 (Fig. 13). As expected, the ratio measured in the linearized DGP simulations, $\overline{\mathfrak{g}}_{\text {vir }}($ lin. DGP, meas $)=1.288 \pm 0.014$ is in excellent agreement with the predicted value of $1+1 /(3 \beta)=1.276$. Similar conclusions hold for the velocity dispersions measured in the sDGP and nDGP-2 simulations, although the results are less constraining due to the smaller force modifications $\left|\overline{\mathfrak{g}}_{\text {vir }}-1\right|$ in those models.

\section{APPLICATION TO OBSERVATIONS}

Observables linked to dynamical masses can be broadly classified into two categories. First, one can measure the velocity distribution of collisionless "tracer particles," such as galaxies within galaxy clusters or stars within galaxies. For a dynamically relaxed system, the kinetic energy $T$ inferred from the velocity distribution is proportional to the potential energy $W$ (Sec. II A), which can be converted into a mass estimate $M_{\Delta \text {,dyn }}$ (we again assume a mass definition in terms of an average interior density $\bar{\rho} \Delta$ ). Several assumptions have to be made in order to obtain the mass estimate. First, one has to assume the galaxies or stars are unbiased tracers of the full matter velocity field (including dark matter). Since member galaxies of a cluster generally reside in overdense substructure (subhalos) of the cluster halo, their velocities might differ systematically from that of the overall matter. Simulation studies $[46,47]$ have shown that this velocity bias is expected to be on the order of $\sim 10 \%$ or less, depending on how galaxies are selected. Further, one has to make assumptions about the density profile shape, and the anisotropy of the velocity distribution, since only the line-of-sight component of the velocity is observed. Nevertheless, our idealized measurement of the dark matter velocity dispersion in the simulations shows that at least in principle, $\sigma_{v}^{2}$ is indeed a good tracer of the modified force $\overline{\mathfrak{g}}_{\text {vir }}$. 
Another set of observations linked to dynamical masses is measurements of the hot ionized gas in galaxy clusters. One technique is to detect the thermal bremsstrahlung in $\mathrm{x}$ rays; another is to measure the upscattering of CMB photons off the hot electrons via the Sunyaev-Zeldovich (SZ) effect. In both techniques, one measures a line-ofsight integral of the electron pressure, with an additional weighting by the electron density in the case of $\mathrm{x}$ rays (since the rate of bremsstrahlung emission is $\propto n_{e} n_{p}=$ $n_{e}^{2}$ ). With some assumptions on the density profile for the baryons, $\mathrm{x}$-ray and SZ signals can be converted into a measurement of the electron pressure as a function of $r$. Instead of the virial theorem that holds for a collisionless system, we now use hydrostatic equilibrium, which is a good assumption at least for dynamically relaxed systems:

$$
\frac{d P}{d r}=\rho_{\text {gas }} \frac{d \Psi}{d r},
$$

where $P$ is the total pressure and $\rho_{\text {gas }}$ is the mass density of the gas, respectively. The difficulty observationally is in measuring the left-hand side of Eq. (62): only the thermal contribution to $P, P_{\text {therm }} \sim n_{\text {gas }} k T$, is directly measurable, while nonthermal contributions from e.g. cosmic rays, bulk flows, and magnetic fields are much harder to estimate. Nevertheless, with appropriate systematic error bars, Eq. (62) is a probe of the gravitational force $d \Psi / d r$.

In summary, a variety of observations lead to estimates of certain weighted averages of the gravitational force,

$$
W_{\mathrm{obs}}=\int d^{3} \mathbf{x} \rho_{\mathrm{obs}}(\mathbf{x}) \mathbf{x} \cdot \nabla \Psi(\mathbf{x}),
$$

where $\rho_{\text {obs }}$ is an effective weight function. In case of x-ray and SZ measurements, it is related to $\rho_{\text {gas }}^{2}$ and $\rho_{\text {gas }}$, respectively, but will be modified by instrumental effects such as the limited instrument aperture. Similarly, for galaxy velocity dispersions in clusters, $\rho_{\text {obs }}$ is proportional to the number density of observed galaxies (again, with observational weights and boundaries folded in).

Now we can use Eq. (10) together with the fact that $\Psi \propto$ $M_{\Delta}^{2 / 3}$, so that $W \propto M^{5 / 3}$ [Eq. (8)]. Then, if the observational mass estimate is done based on ordinary gravity, so that in GR the mass estimate equals the true mass $M_{\Delta}$, the resulting mass estimate $M_{\Delta \text {,dyn }}$ in modified gravity is, in fact,

$$
M_{\Delta, \mathrm{dyn}}=\overline{\mathrm{g}}_{\mathrm{obs}}^{3 / 5} M_{\Delta} .
$$

Here $\overline{\mathfrak{g}}_{\text {obs }}$ is a weighted integral using Eq. (11), with $\rho$ replaced by the effective weight $\rho_{\text {obs }}$. Note that in general $\overline{\mathfrak{g}}_{\text {obs }}$ will depend on the true mass $M_{\Delta}$ itself.

Since the true mass can in principle be obtained from weak or strong lensing, a comparison of lensing mass with the dynamical mass Eq. (64) can be used to measure the modified forces in $f(R)$ or DGP. Again, it is important to take into account the unavoidable observational weighting that is being done in the measurements of both $M_{\Delta \text {,dyn }}$ and $M_{\Delta}$.

Recently, the SLACS sample of elliptical galaxies acting as strong lenses [48] has been used to constrain deviations from GR [28,49]. Furthermore, using a similar argument as the thin-shell condition Eq. (36) (Sec. II C), [49] has shown that these measurements constrain the $f(R)$ model considered here at the level of $\left|f_{R 0}\right| \lesssim 2 \times 10^{-6}$. For these constraints one has to make some assumptions on the potential well of the lens galaxy, for example, that it is dominated by the density distribution of the inner few kpc, thus neglecting any larger scale potential well. As we have seen, the magnitude of the force modification in $f(R)$ can depend somewhat on the environment. In particular, we found that a subset of halos around 3-8 $\times 10^{13} M_{\odot} / h$ (at the low-mass end of the range accessible to the simulations) is screened much more strongly than expected for isolated halos, consistent with an effect of the large-scale environment. Nevertheless, strong lens galaxies offer a quite powerful probe of gravity on kpc scales, if the environmental effects can be understood.

On larger scales, the comparison of dynamical and lensing masses of massive galaxy clusters can be interesting since they dominate their local environment, so that environmental effects should be negligible. Also, for cluster-scale masses we were able to validate our theoretical models for $\overline{\mathrm{g}}_{\text {vir }}$ directly with the modified gravity simulations (Sec. III). However, for clusters it is preferable to measure the dynamics and lensing at large scales: first, the deviations from GR quickly shrink close to the cluster core owing to the chameleon and Vainshtein mechanisms; second, baryonic effects on the observables and the density profile, such as cooling and active galactic nuclei feedback, are expected to be less significant at greater distances from the cluster center.

It is also possible to use dynamic mass estimates of clusters by themselves, without direct comparison to lensing masses. As shown in [50-55], the abundance of massive clusters is a sensitive probe of the growth of structure as well as gravity. When comparing the observed cluster mass function measured using a dynamical mass measure with modified gravity predictions, it is necessary to take into account the effect of the modified forces on the mass estimates as well. In order to estimate the effect on the observed mass function, we use Eq. (64), setting $\overline{\mathfrak{g}}_{\text {obs }}=$ $\overline{\mathrm{g}}_{\text {vir }}$, the idealized quantity we have modeled and calibrated with simulations. Dynamical mass measures (i.e. velocity dispersions) in our simulations are noisy (Sec. III); thus we have simply rescaled the mass of each halo in the modified gravity simulations by our theoretical model of $\overline{\mathfrak{g}}_{\text {vir }}(M)$ for the given cosmology.

Figure 15 shows the relative enhancement of the mass function in $f(R)$ gravity with respect to $\Lambda \mathrm{CDM}$, when measured using lensing masses (i.e. true $M_{300}$ ) and dy- 


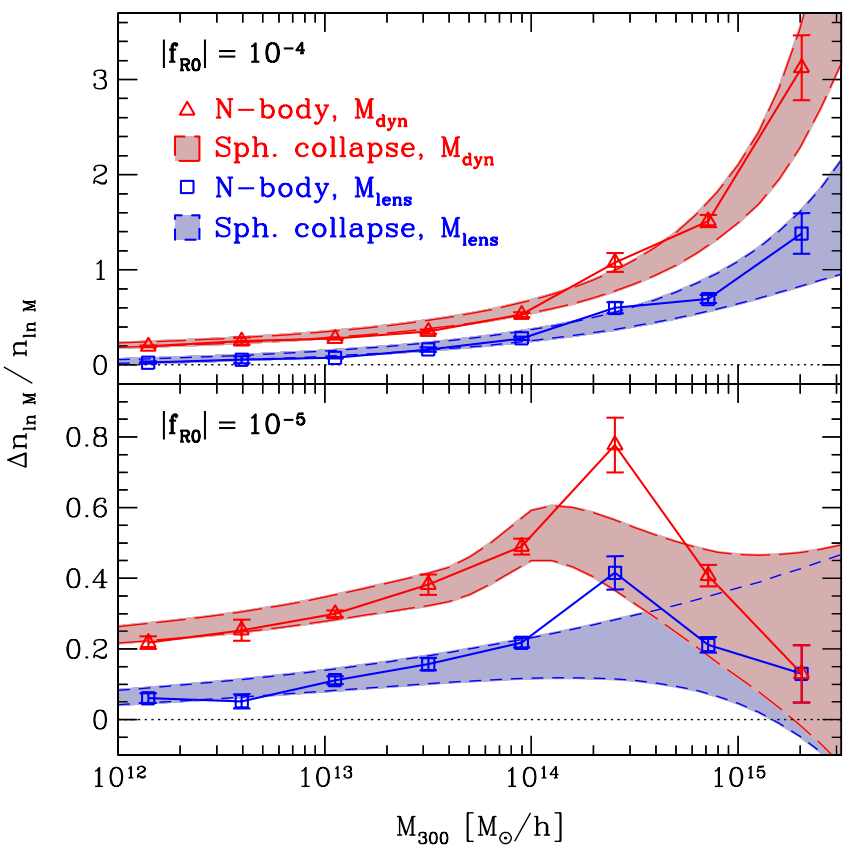

FIG. 15 (color online). Mass function enhancement in $f(R)$ relative to a $\Lambda \mathrm{CDM}$ cosmology with the same expansion history, $n_{\ln M}(f(R)) / n_{\ln M}(\Lambda \mathrm{CDM})-1$, for $\left|f_{R 0}\right|=10^{-4}$ (top panel) and $10^{-5}$ (bottom panel). The points show simulation results, while the shaded bands show spherical collapse predictions [32] (see text). Results are shown for the mass function $n_{\ln M_{\Delta}}$ in terms of the lensing mass and $n_{\ln M_{\Delta, \text { dyn }}}$ in terms of the dynamical mass (see text; $\Delta=300$ in both cases).

namical masses, for ${ }^{4}\left|f_{R 0}\right|=10^{-4}$ and $10^{-5}$. Clearly, the observed abundance of halos is further enhanced when measured in terms of dynamical masses. In the mass range where halos are unscreened, the mass function enhancement is boosted by a factor of 2 or more. This is because the dynamical mass estimate is a factor of $(4 / 3)^{3 / 5} \approx 1.19$ higher than the lensing mass in $f(R)$ gravity in the unscreened case, in conjunction with the steeply falling mass function. Constraints on $f(R)$ gravity from x-ray clusters could thus be significantly improved by using the dynamical mass function instead of the true or lensing mass function that was used in [54]. Note the sharp turnover in the mass function enhancement for the intermediate field value. This transition due to the onset of the chameleon mechanism is already present in the lensing mass function [32]. Since $\overline{\mathfrak{g}}_{\text {vir }}$ transitions from $4 / 3$ to 1 in this mass range as well, the effect is enhanced in the dynamical mass function.

The shaded bands in Fig. 15 show the spherical collapse predictions presented in [32]. These are based on the linear $f(R)$ matter power spectrum together with the Sheth-

\footnotetext{
${ }^{4}$ Since all halos above $\sim 10^{13} M_{\odot} / h$ are screened for the small field $\left|f_{R 0}\right|=10^{-6}$, the dynamical mass function is essentially equal to the lensing mass function for most of the mass range and is not repeated here (see [32])
}

Tormen prescription, using two sets of collapse parameters derived for limiting cases of spherical collapse in $f(R)$ (enhanced forces throughout, and unmodified forces). We rescaled the predictions in terms of lensing mass given in [32] to the dynamical mass via

$$
n_{\ln M_{\Delta, \mathrm{dyn}}} \equiv \frac{d n}{d \ln M_{\Delta, \mathrm{dyn}}}=\frac{d \ln M_{\Delta}}{d \ln M_{\Delta, \mathrm{dyn}}} n_{\mathrm{ln} M_{\Delta}} .
$$

As expected, the predictions in terms of dynamical mass perform equally well as those for the lensing mass. Since our prediction for $\overline{\mathrm{g}}_{\mathrm{vir}}$ includes the chameleon mechanism, the predictions for the intermediate field value show a corresponding transition at approximately the right mass. Still, the predictions do not match the simulation results completely due to the shortcomings of our simple spherical collapse model [32].

Figure 16 shows the corresponding results for the two normal-branch DGP models nDGP-1 and nDGP-2. The effect is less dramatic on the DGP mass function, since $\overline{\mathrm{g}}_{\text {vir }}$ in DGP is generally smaller than in $f(R)$. Nevertheless, the impact, especially for nDGP-1, is significant, implying an abundance boost of $\sim 50 \%$ at high masses. The shaded bands in Fig. 16 again show a spherical collapse model [41], which uses the analytical solution for the modified forces in DGP in the spherically symmetric case as one limiting case of spherical collapse in DGP. The other limit is given by using the linearized expression for the modified forces. Again, the spherical collapse model performs

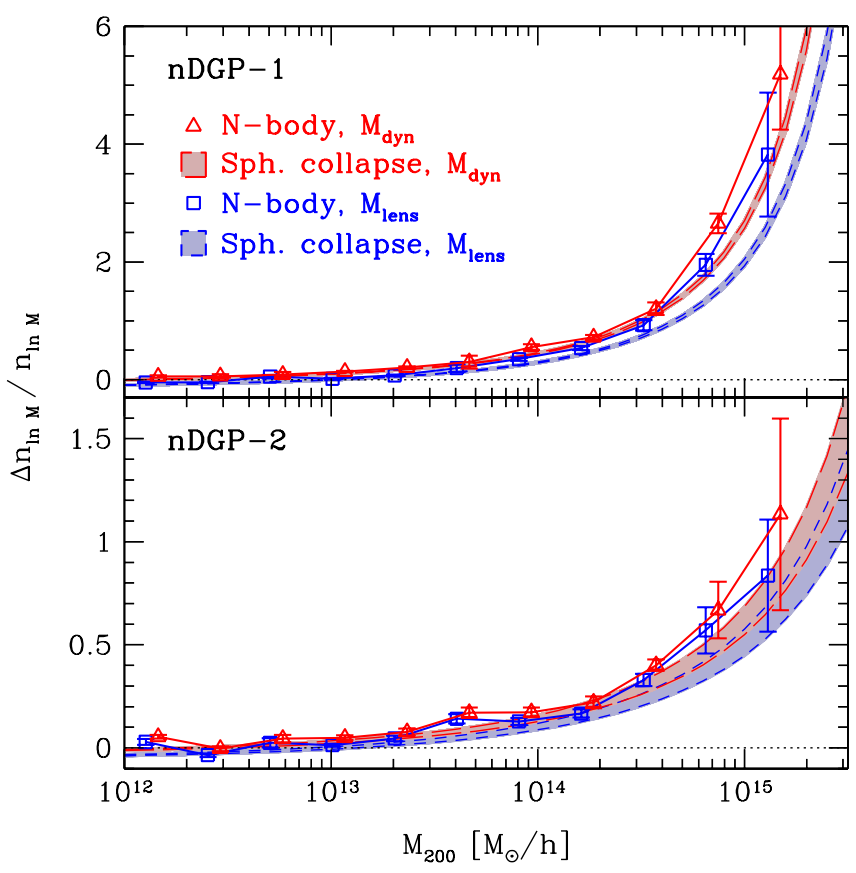

FIG. 16 (color online). Same as Fig. 15, but for the DGP models nDGP-1 (top panel) and nDGP-2 (bottom panel). The simulation results for the dynamical mass (red triangles) have been displaced horizontally for clarity. The shaded band shows the spherical collapse model of [41]. 
equally well for the mass function in terms of dynamical mass as for the lensing mass function.

\section{CONCLUSIONS}

In this paper, we have studied the dynamics of matter within bound cosmic structures, i.e. dark matter halos, in $f(R)$ and DGP. The potential governing matter dynamics can differ from the lensing potential by $20 \%-30 \%$ in these models. These unique signatures of modified gravity can be observed by comparing dynamical and lensing mass estimates of clusters or galaxies. Furthermore, they strongly influence the observed abundance of massive clusters when measured via dynamical mass proxies such as $\mathrm{x}$ rays or the $\mathrm{SZ}$ effect. For example, the enhancement of the cluster abundance in $f(R)$ (with respect to $\Lambda \mathrm{CDM}$ ) at a fixed dynamical mass can be roughly twice that measured if the mass is based on lensing measurements. These signatures in the dynamics are also relevant for large-scale structure observations, such as the redshift-space power spectrum or correlation function on small scales.

However, since halos are highly nonlinear objects, the peculiar chameleon and Vainshtein mechanisms play a crucial role, as they are necessary in order to restore general relativity in high-density environments. Thus, the dynamics in these models can only be rigorously studied through $N$-body simulations that include the nonlinear mechanisms of $f(R)$ and DGP consistently.

In the case of $f(R)$, the chameleon mechanism is triggered once the depth of the potential well is comparable to the background value of the scalar field. The suppression of the force modifications within a halo thus depends not only on the halo mass but also its environment. Consequently, we found significant scatter from halo to halo in the force modification $g$ measured in the $f(R)$ simulations. Furthermore, we identified a subset of halos which are in the close vicinity of massive neighbors, and which show a much stronger suppression of the force modifications than expected for isolated halos. In the majority of cases, however, the simulation results confirm the basic expectation that halos are "unscreened" below a certain threshold mass determined by the potential well and the field value, whereas GR is restored at higher masses. Furthermore, a simple model based on the spherically symmetric solution of the field equations provides a good match to the scale as well as mass dependence of the force modifications in $f(R)$.

In DGP, the nonlinear suppression of the force modifications through the Vainshtein mechanism is much less dependent on halo mass and details of the large-scale environment. Instead, the crucial quantity is the average mass density within a given radius. Thus, uncertainties in the semianalytic predictions for DGP are mainly due to the density profile and are already quite small. When taking into account the force resolution of the simulations, our predictions provide an excellent fit to the simulation measurements. Since the basic assumptions of the model, in particular, spherical symmetry, seem to hold well, we expect that force modifications can be predicted very accurately in DGP, provided the density profile is known sufficiently well.

Given that our semianalytic models appear to capture the mass and scale dependence of the modified forces correctly for both $f(R)$ and DGP, they can be useful in extending predictions beyond the limits of resolution and parameter space of the simulations. This will be necessary, in particular, for the comparison with observations.

While this study is specific to $f(R)$ and DGP, it shows the qualitative features expected in observations of dynamics from viable modified gravity models, which employ a nonlinear mechanism to restore GR locally. In the outer regions of massive clusters, as well as in lower mass objects, these models generally predict order unity deviations from GR. Observations in this regime thus offer the perspective of closing the last remaining loopholes for significant modifications to gravity on large scales.

\section{ACKNOWLEDGMENTS}

I would like to thank Wayne Hu, Tristan Smith, Mark Wyman, and Donghai Zhao for discussions and comments on the paper. This work was supported by the Gordon and Betty Moore Foundation at Caltech. The simulations used in this work have been performed on the Joint FermilabKICP Supercomputing Cluster, supported by grants from Fermilab, Kavli Institute for Cosmological Physics, and the University of Chicago.
[1] P. Zhang, M. Liguori, R. Bean, and S. Dodelson, arXiv:0704.1932.

[2] B. Jain and P. Zhang, Phys. Rev. Lett. 99, 141302 (2008).

[3] Y.-S. Song and K. Koyama, J. Cosmol. Astropart. Phys. 01 (2009) 048.

[4] L. Knox, Y.-S. Song, and J. A. Tyson, Phys. Rev. D 74, 023512 (2006).
[5] F. Schmidt, Phys. Rev. D 78, 043002 (2008).

[6] Y.-S. Song, arXiv:astro-ph/0602598.

[7] S. Tsujikawa and T. Tatekawa, Phys. Lett. B 665, 325 (2008).

[8] P. Zhang, Phys. Rev. D 73, 123504 (2006).

[9] Y.-S. Song, I. Sawicki, and W. Hu, Phys. Rev. D 75, 064003 (2007). 
[10] F. Schmidt, M. Liguori, and S. Dodelson, Phys. Rev. D 76, 083518 (2007).

[11] J. Uzan, arXiv:0908.2243.

[12] S. M. Carroll, V. Duvvuri, M. Trodden, and M. S. Turner, Phys. Rev. D 70, 043528 (2004).

[13] S. Nojiri and S.D. Odintsov, Phys. Rev. D 68, 123512 (2003).

[14] S. Capozziello, S. Carloni, and A. Troisi, Recent Res. Dev. Astron. Astrophys. 1, 625 (2003).

[15] T. P. Sotiriou and V. Faraoni, Rev. Mod. Phys. 82, 451 (2010).

[16] G. Dvali, G. Gabadadze, and M. Porrati, Phys. Lett. B 485, 208 (2000).

[17] H. Oyaizu, Phys. Rev. D 78, 123523 (2008).

[18] F. Schmidt, Phys. Rev. D 80, 043001 (2009).

[19] K. C. Chan and R. Scoccimarro, Phys. Rev. D 80, 104005 (2009).

[20] F. Schmidt, Phys. Rev. D 80, 123003 (2009).

[21] J. Khoury and M. Wyman, Phys. Rev. D 80, 064023 (2009).

[22] J. D. Bekenstein and R. H. Sanders, Astrophys. J. 429, 480 (1994).

[23] R. Scoccimarro, Phys. Rev. D 70, 083007 (2004).

[24] P. Zhang, M. Liguori, R. Bean, and S. Dodelson, Phys. Rev. Lett. 99, 141302 (2007).

[25] J. A. Peacock and S. J. Dodds, Mon. Not. R. Astron. Soc. 280, L19 (1996).

[26] J. F. Navarro, C. S. Frenk, and S. D. M. White, Astrophys. J. 490, 493 (1997).

[27] C. M. Will, Living Rev. Relativity 9, 3 (2005).

[28] J. Schwab, A. S. Bolton, and S. A. Rappaport, Astrophys. J. 708, 750 (2010).

[29] L.D. Shaw, J. Weller, J.P. Ostriker, and P. Bode, Astrophys. J. 646, 815 (2006).

[30] A. J. Cuesta, F. Prada, A. Klypin, and M. Moles, Mon. Not. R. Astron. Soc. 389, 385 (2008).

[31] A. E. Evrard et al., Astrophys. J. 672, 122 (2008).

[32] F. Schmidt, M. Lima, H. Oyaizu, and W. Hu, Phys. Rev. D 79, 083518 (2009).

[33] J. S. Bullock, T. S. Kolatt, Y. Sigad, R. S. Somerville, A. V. Kravtsov, A. A. Klypin, J. R. Primack, and A. Dekel, Mon. Not. R. Astron. Soc.321, 559 (2001).
[34] A. V. Macciò, A. A. Dutton, F. C. van den Bosch, B. Moore, D. Potter, and J. Stadel, Mon. Not. R. Astron. Soc. 378, 55 (2007).

[35] D. H. Zhao, Y. P. Jing, H. J. Mo, and G. Börner, Astrophys. J. 707, 354 (2009).

[36] D. H. Zhao, Y. P. Jing, H. J. Mo, and G. Börner, Astrophys. J. Lett. 597, L9 (2003).

[37] W. Hu and I. Sawicki, Phys. Rev. D 76, 064004 (2007).

[38] J. Khoury and A. Weltman, Phys. Rev. D 69, 044026 (2004).

[39] H. Oyaizu, M. Lima, and W. Hu, Phys. Rev. D 78, 123524 (2008).

[40] C. Deffayet, Phys. Lett. B 502, 199 (2001).

[41] F. Schmidt, W. Hu, and M. Lima, Phys. Rev. D 81, 063005 (2010).

[42] W. Fang, S. Wang, W. Hu, Z. Haiman, L. Hui, and M. May, Phys. Rev. D 78, 103509 (2008).

[43] A. Nicolis and R. Rattazzi, J. High Energy Phys. 6 (2004) 059.

[44] K. Koyama and F. P. Silva, Phys. Rev. D 75, 084040 (2007).

[45] A. Lue, R. Scoccimarro, and G. D. Starkman, Phys. Rev. D 69, 124015 (2004).

[46] A. Faltenbacher and J. Diemand, Mon. Not. R. Astron. Soc. 369, 1698 (2006).

[47] E. T. Lau, D. Nagai, and A. V. Kravtsov, Astrophys. J. 708, 1419 (2010).

[48] A. S. Bolton, S. Burles, L. V.E. Koopmans, T. Treu, and L. A. Moustakas, Astrophys. J. 638, 703 (2006).

[49] T. L. Smith, arXiv:0907.4829.

[50] S.D. M. White, J.F. Navarro, A.E. Evrard, and C.S. Frenk, Nature (London) 366, 429 (1993).

[51] V. R. Eke, S. Cole, C. S. Frenk, and J. Patrick Henry, Mon. Not. R. Astron. Soc. 298, 1145 (1998).

[52] S. Borgani, P. Rosati, P. Tozzi, S. A. Stanford, P. R. Eisenhardt, C. Lidman, B. Holden, R. Della Ceca, C. Norman, and G. Squires, Astrophys. J. 561, 13 (2001).

[53] A. Vikhlinin et al., Astrophys. J. 692, 1060 (2009).

[54] F. Schmidt, A. Vikhlinin, and W. Hu, Phys. Rev. D 80, 083505 (2009).

[55] A. Mantz, S. W. Allen, D. Rapetti, and H. Ebeling, arXiv:0909.3098. 